\title{
Sequential Weak Approximation for Maps of Finite Hessian Energy
}

\author{
Robert Hardt*and Tristan Rivière ${ }^{\dagger}$
}

June 19, 2018

\begin{abstract}
Consider the space $W^{2,2}(\Omega ; N)$ of second order Sobolev mappings $v$ from a smooth domain $\Omega \subset \mathbb{R}^{m}$ to a compact Riemannian manifold $N$ whose Hessian energy $\int_{\Omega}\left|\nabla^{2} v\right|^{2} d x$ is finite. Here we are interested in relations between the topology of $N$ and the $W^{2,2}$ strong or weak approximability of a $W^{2,2}$ map by a sequence of smooth maps from $\Omega$ to $N$. We treat in detail $W^{2,2}\left(\mathbb{B}^{5}, \mathbb{S}^{3}\right)$ where we establish the sequential weak $W^{2,2}$ density of $W^{2,2}\left(\mathbb{B}^{5}, \mathbb{S}^{3}\right) \cap \mathcal{C}^{\infty}$. The strong $W^{2,2}$ approximability of higher order Sobolev maps has been studied in the recent preprint [BPV] of P. Bousquet, A. Ponce, and J. Van Schaftigen. For an individual map $v \in W^{2,2}\left(\mathbb{B}^{5}, \mathbb{S}^{3}\right)$, we define a number $L(v)$ which is approximately the total length required to connect the isolated singularities of a strong approximation $u$ of $v$ either to each other or to $\partial \mathbb{B}^{5}$. Then $L(v)=0$ if and only if $v$ admits $W^{2,2}$ strongly approximable by smooth maps. Our critical result, obtained by constructing specific curves connecting the singularities of $u$, is the bound $L(u) \leq c \int_{\mathbb{R}_{5}}\left|\nabla^{2} u\right|^{2} d x$. This allows us to construct, for the given Sobolev map $v \in W^{2,2}\left(\mathbb{B}^{5}, \mathbb{S}^{3}\right)$, the desired $W^{2,2}$ weakly approximating sequence of smooth maps. To find suitable connecting curves for $u$, one uses the twisting of a $u$ pull-back normal framing of a suitable level surface of $u$.
\end{abstract}

Math. Class. 58D15, 46E35, 49Q99.

\section{Contents}

I Introduction 2

I.1 Strong Approximation . . . . . . . . . . . . . . . . . . . . . . . . . 2

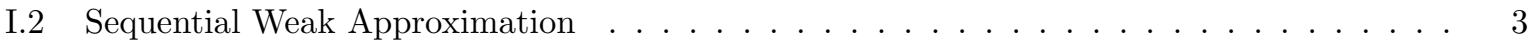

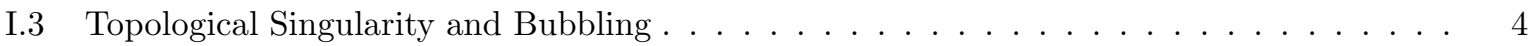

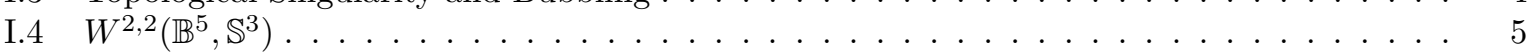

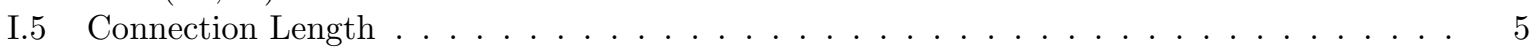

II Preliminaries 6

III A Strongly Dense Family with Isolated Singularities 8

III.1 Strong Approximation by Maps in $\mathcal{R} \ldots \ldots \ldots \ldots \ldots \ldots$

III.2 Insertion of an $\mathbb{S H H}$ Bubble into a Map from $\mathbb{B}^{4}$ to $\mathbb{S}^{3} \ldots \ldots \ldots \ldots \ldots \ldots$

III.3 Singularity Cancellation of a Map in $\mathcal{R} \ldots \ldots \ldots \ldots$. . . . . . . . . . . . 12

*Department of Mathematics, Rice University, Houston, TX 77251, USA. Research partially supported by the NSF.

${ }^{\dagger}$ Forschungsinstitut für Mathematik, ETH Zentrum, CH-8093 Zürich, Switzerland. 
IV Connecting Singularities with Controlled Length 16

IV.1 Estimates for Choosing the Level Surface $\Sigma=u^{-1}\{p\} \ldots \ldots \ldots \ldots \ldots \ldots$. . . . . . . . . . . . . . .

IV.2 A Pull-back Normal Framing for $\Sigma=u^{-1}\{p\} \ldots \ldots \ldots \ldots \ldots$. . . . . . . . . 17

IV.3 Twisting of the Normal Frame $\tilde{\tau}_{1}, \tilde{\tau}_{2}, \tilde{\tau}_{3}$ About Each Singularity $a_{i} \ldots \ldots \ldots \ldots \ldots$

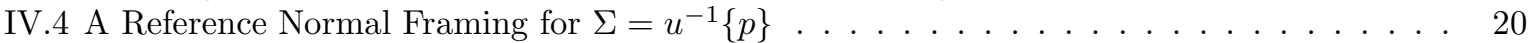

IV.5 Asymptotic Behavior of $\gamma$ Near the Singularities $a_{i}$ and $b_{j} \ldots \ldots \ldots \ldots \ldots \ldots . \ldots \ldots$

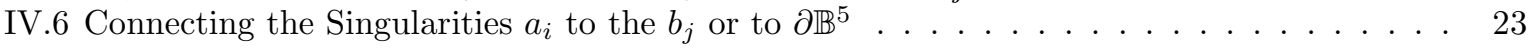

IV.7 Estimating the Length of the Connecting Set $A \ldots \ldots \ldots \ldots \ldots \ldots \ldots$

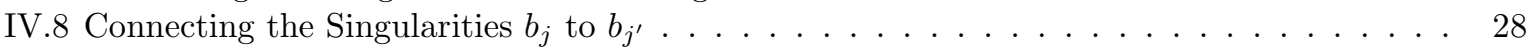

IV.9 Estimating the Length of the Connecting Set $B \ldots \ldots \ldots \ldots \ldots \ldots$

V Sequential Weak Density of $W^{2,2}\left(\mathbb{B}^{5}, \mathbb{S}^{3}\right)$

V.1 Least Connection Length $L(v) \ldots \ldots \ldots \ldots \ldots \ldots \ldots \ldots \ldots$

\section{Introduction}

To motivate our specific work on weak sequential approximability of $W^{2,2}$ maps from $\mathbb{B}^{5}$ to $\mathbb{S}^{3}$, we will first describe briefly the background and general problems. Let $(N, g)$ be a compact Riemannian manifold. Via the Nash embedding theorem, one may assume that $N$ is a submanifold of some Euclidian space $\mathbb{R}^{\ell}$ and that the metric $g$ is induced by this inclusion. One then has, for any open subset $\Omega$ of $\mathbb{R}^{m}, k \in \mathbb{N}$, and $p>1$, the nonlinear space of $k$ th order, Sobolev maps

$$
W^{k, p}(\Omega, N)=\left\{u \in W^{k, p}\left(\Omega, \mathbb{R}^{\ell}\right): u(x) \in N \text { for almost every } \mathrm{x} \in \Omega\right\},
$$

where $W^{k, p}\left(\Omega, \mathbb{R}^{\ell}\right)$ denotes the Banach space of $\mathbb{R}^{\ell}$-valued, order $k$, Sobolev functions on $\Omega$ with norm $\|u\|_{W^{k, p}}=\left[\sum_{j=0}^{k}\left(\int_{\Omega}\left|\nabla^{j} u\right|^{p} d x\right)^{2 / p}\right]^{1 / 2}$.

\section{I.1 Strong Approximation}

A basic question concerning the spaces $W^{k, p}(\Omega, N)$ is the approximability of these maps by a sequence of smooth maps of $\Omega$ into $N$. The issue involves the possible discontinuities in a Sobolev map because any continuous Sobolev map may be approximated strongly in the Sobolev norm. In fact, here ordinary smoothing [A] gives both uniform and $W^{2,2}$ strong approximation by an $\mathbb{R}^{\ell}$-valued smooth Sobolev function whose image lies in a small neighborhood of $N$; then composing this with the nearest-point projection to $N$ gives the desired smooth strong approximation with image in $N$. It was first observed in SU] that for $n=2$, a $W^{1,2}$ map (which may fail to have a continuous representative) admits strong $W^{1,2}$ approximation by smooth $W^{1,2}$ maps into $N$. However for $n=3$, [SU] also showed that the specific singular Sobolev map $x /|x| \in W^{1,2}\left(\mathbb{B}^{3}, \mathbb{S}^{2}\right)$ is not strongly approximable in $W^{1,2}$ by smooth maps from $\mathbb{B}^{3}$ to $\mathbb{S}^{2}$. For first order Sobolev maps, the general problem of strong $W^{1, p}$ approximability was treated by F. Bethuel in [Be2], which (with [BZ]) shows that:

$$
W^{1, p}\left(\mathbb{B}^{m}, N\right) \text { is the sequential strong } W^{1, p} \text { closure of } \mathcal{C}^{\infty}\left(\mathbb{B}^{m}, N\right) \quad \Longleftrightarrow \quad \Pi_{[p]}(N)=0 .
$$

Here $[p]$ is the greatest integer less than or equal to $p$. F. Hang and F. H. Lin, in HaL1] and HaL2, updated these results with some new proofs and corrections, which account for the role played by the topology of the domain in approximability questions. See HaL2, Th.1.3 for the precise conditions on the domain. There are many other interesting works on strong approximability of first order Sobolev maps by smooth maps, e.g. $[\mathrm{Be1}], \overline{\mathrm{BCL}}, \overline{\mathrm{Hj}}], \overline{\mathrm{BCDH}}, \overline{\mathrm{BBC}},[\mathrm{BZ}]$. Generalization of the strong approximability 
results of [Be2], HaL1], and HaL2] to higher order Sobolev mappings has been treated by P. Bousquet, A. Ponce, and J. Van Schaftigen in BPV] which (with [BZ]) shows that

$$
W^{k, p}\left(\mathbb{B}^{m}, N\right) \text { is the sequential strong } W^{k, p} \text { closure of } \mathcal{C}^{\infty}\left(\mathbb{B}^{m}, N\right) \quad \Longleftrightarrow \quad \Pi_{[k p]}(N)=0 .
$$

\section{I.2 Sequential Weak Approximation}

The space $W^{k, p}(\Omega, N)$ also inherits the weak topology from $W^{k, p}\left(\Omega, \mathbb{R}^{\ell}\right)$. A Sobolev map in $W^{k, p}(\Omega, N)$ that is not $W^{k, p}$ strongly approximable by smooth maps may be $W^{k, p}$ weakly approximable by a sequence of smooth maps. For example, the map $x /|x| \in W^{1,2}\left(\mathbb{B}^{3}, \mathbb{S}^{2}\right)$ is weakly approximable in $W^{1,2}$ by some sequence $u_{i}$ of smooth maps. The well-known construction of such a $u_{i}$ involves changing $x /|x|$ in a thin cylindrical tunnel $U$ of width $1 / i$ extending from the origin $(0,0,0)$ to a point on $\partial \mathbb{B}^{3}$. To prove the weak $W^{1,2}$ weak convergence of $u_{i}$ to $x /|x|$, the key point of the construction is to keep the energies $\int_{\mathbb{B}^{3}}\left|\nabla u_{i}\right|^{2} d x$ bounded independent of $i$.

To find an example of a map $v \in W^{1, p}\left(\mathbb{B}^{m}, N\right)$ which does not have a weakly approximating sequence of smooth maps, we need both $p<m$ and $\Pi_{[p]}(N) \neq 0$. Then, if $p$ is not an integer, we simply choose, as in $\mathrm{Be} 2$, any map $v$ which fails to have strong smooth approximations. Assuming for contradiction that this map $v$ did admit some weak approximation by smooth maps $v_{i}$, then, for every point $a \in \mathbb{B}^{m}$, Fubini's theorem and Sobolev embedding (because $p>[p]$ ), would give strong convergence of the restrictions $v_{i} \mid \mathbb{S}_{a}$ to $v \mid \mathbb{S}_{a}$ for almost every $[p]$ dimensional Euclidean sphere $\mathbb{S}_{a}$ centered at $a$ in $\mathbb{B}^{m}$. Then by the smoothness of $v_{i}$ and by $[\mathrm{W}]$, the corresponding homotopy classes $\llbracket v \mid \mathbb{S}_{a} \rrbracket$ would all vanish. But Bethuel showed in [Be1] that precisely this local vanishing homotopy condition on $[p]$ spheres would imply that $v$ does admit strong smooth approximation, a contradiction.

For integer $p$ the following question is still open:

For any any compact manifold $N$, any integers $k, m \geq 1$ and any integer $p \geq 2$, is every Sobolev map $v \in W^{k, p}\left(\mathbb{B}^{m}, N\right)$ actually $W^{k, p}$ weakly approximable by a sequence of smooth maps?

This sequential weak density of smooth maps has been verified in the following cases:

(1) $\mathrm{BBC}$, $\mathrm{ABL}: W^{1, p}\left(\mathbb{B}^{m}, \mathbb{S}^{p}\right)$.

(2) $\mathrm{Hj}: W^{1, p}\left(\mathbb{B}^{m}, N\right)$ with $N$ being simply $p-1$ connected (i.e. $\Pi_{j}(N)=0$ for $0 \leq j \leq p-1$ ).

(3) $\left[\mathrm{Pa}\right.$ : $W^{1,1}(M, N)$ with $M$ and $N$ being arbitrary smooth manifolds with $\partial N=\emptyset$ (weak convergence has to be understood in a biting sense here)

(4) $[\mathrm{PR}]: W^{1,2}\left(\mathbb{B}^{m}, N\right)$. (See also [Ha] concerning the role of the topology of $M$ in $W^{1,2}(M, N)$.)

See also a presentation of these results in $\mathrm{Ri}$. Another case is the main result of the present paper:

Theorem V] Any map in $W^{2,2}\left(\mathbb{B}^{5}, \mathbb{S}^{3}\right)$ may be approximated in the $W^{2,2}$ weak topology by a sequence of smooth maps.

In $₫$ I.4 below, we will explain how we came to study maps from $\mathbb{B}^{5}$ to $\mathbb{S}^{3}$ and to look for $W^{2,2}$ estimates. But first we review a few of the ideas that were developed to study sequential weak convergence of smooth maps. The space $W^{1,2}\left(\mathbb{B}^{3}, \mathbb{S}^{2}\right)$ was studied extensively in the late eighties and early nineties with many works, e.g. [HL], BCL, [BZ], [BBC], GMS1. The concrete results of these many works has led to some analogous results and many conjectures for more general $k, n, p$, and $N$. To sequentially weakly approximate a map $v \in W^{1,2}\left(\mathbb{B}^{3}, \mathbb{S}^{2}\right)$, one first finds a $W^{1,2}$ strong approximation from the family $\mathcal{R}_{0}\left(\mathbb{B}^{3}, \mathbb{S}^{2}\right)$ of maps $u \in W^{1,2}\left(\mathbb{B}^{3}, \mathbb{S}^{2}\right)$ which are smooth away from some finite set Sing $u$. In particular, we may assume $\int_{\mathbb{B}^{3}}|\nabla u|^{2} d x \leq 2 \int_{\mathbb{B}^{3}}|\nabla v|^{2} d x$. Here the topology of $u$ near a point $a \in \operatorname{Sing} v$ is given by the integer $d(a)=$ degree $\left[u \mid \partial \mathbb{B}_{\varepsilon}(a)\right]$, which is independent of a.e. small $\varepsilon$. Then to get the desired completely smooth weak approximate, it is necessary to essential cancel the singularities of $u$. One does this by finding a one-chain or "connection" $\Gamma_{u}$ with $\partial \Gamma_{u}$ in $\mathbb{B}^{3}$ being $\sum_{a \in \operatorname{Sing} v} d(a) \llbracket a \rrbracket$ and with the rest of $\partial \gamma_{u}$ 
lying in $\partial B^{3}$. Then, as with the argument for $x /|x|$, one constructs smooth maps $u_{i}$ by making changes in tunnels of radius $1 / i$ centered along the connection. To keep the $\left|\nabla u_{i}\right|^{2}$ integrals bounded, one needs to find a bound for the total length of the connection $\Gamma_{u}$ that depends only on $v$, and is independent of the approximating $u$. Here one may find a suitable connection by using the coarea formula. This gives a good level curve of $u$ which connects the singularities to each other and to $\partial \mathbb{B}^{3}$ and which has length bounded by $\int_{\mathbb{B}^{3}}|\nabla u|^{2} d x$, which has the independent bound $2 \int_{\mathbb{B}^{3}}|\nabla v|^{2} d x$.

The first part of this argument, the strong $W^{1,2}$ approximation of an arbitrary Sobolev map $v \in$ $W^{1,2}\left(\mathbb{B}^{3}, \mathbb{S} 2\right)$ by a map $u \in \mathcal{R}_{0}\left(\mathbb{B}^{3}, \mathbb{S}^{2}\right)$ has been generalized in $\mathrm{Be} 2$ to all $W^{1, p}\left(\mathbb{B}^{m}, N\right)$ and recently in $\left[\mathrm{BPV}\right.$ to all $W^{k, p}\left(\mathbb{B}^{m}, N\right)$. Here one gets strong approximation by maps in $\mathcal{R}_{m-[p]-1}\left(\mathbb{B}^{m}, N\right)$ (respectively, $\mathcal{R}_{m-[k p]-1}\left(\mathbb{B}^{m}, N\right)$ which are smooth with singularities lying in finitely many affine planes of dimension $m-[p]-1$ (respectively, $m-[k p]-1$ ).

However, the second part involving canceling the singularities of $u$ has proven very challenging for generalization. One roughly needs an $m-[p]$ (respectively, $m-[k p]-1$ ) dimensional connection which has mass bounded in terms of the energy of $u$ and which the connects the singularity. Even with the connection, one still has to construct the bounded energy, smooth approximate.

\section{I.3 Topological Singularity and Bubbling}

In this supercritical dimension $m>k p$, we see that studying sequential $W^{k, p}$ weak smooth approximation in $W^{k, p}\left(\mathbb{B}^{m}, N\right)$ leads to questions about the relationship between the possible energy drop, $\int_{\mathbb{B}^{m}}\left|\nabla^{k} u\right|^{p} d x<\liminf _{i \rightarrow \infty} \int_{\mathbb{B}^{m}}\left|\nabla^{k} u_{i}\right|^{p} d x$, of a $W^{k, p}$ weakly convergent sequence $u_{i} \in W^{k, p}\left(\mathbb{B}^{m}, N\right) \cap \mathcal{C}^{\infty}$ and the possible singularities of its weakly convergent limit $u \in W^{k, p}\left(\mathbb{B}^{m}, N\right)$.

For $0 \neq \alpha \in \Pi_{k p}(N)$, we say a point $a \in \mathbb{B}^{m}$ is a type $\alpha$ topological singularity of a $W^{k, p}$ map $u$ if there is an $k p+1$ dimensional affine plane $P$ containing $a$ so the restrictions of $u$ to a.e. small $k p$ sphere $P \cap \partial \mathbb{B}_{\varepsilon}(a)$ induce (i.e. in the sense of $[\mathrm{W}]$ ) the homotopy class $\alpha$. Following the $W^{k, p}$ strong density of the partially smooth maps $\mathcal{R}_{m-k p-1}\left(\mathbb{B}^{m}, N\right)$ in $W^{k, p}\left(\mathbb{B}^{m}, N\right)$, one expects the topological singularities, with their types as coefficients, to form a chain $S_{u}$ having dimension $m-k p-1$ and having coefficients in the group $\Pi_{k p}(N)$. Recall the criterion of [Be1] that the vanishing of this " $u$ topological singularity" chain( that is the vanishing of such homotopy classes for a.e. such restrictions at every $a \in \mathbb{B}^{m}$ ) is equivalent the $W^{k, p}$ strong approximability of $u$ by smooth maps.

Also for $0 \neq \alpha \in \Pi_{k p}(N)$, the restrictions of $u_{i}$ to generic affine $k p$ planes can, as $i \rightarrow \infty$ have $\left|\nabla^{k}\right|^{p}$ energy concentration at an isolated point $b$ with an associated topological change corresponding to a type beta "bubble". Putting such points together with their bubble types as coefficients should give a " $u_{i}$ bubbled" chain $B_{u_{i}}$ that has dimension $m-k p$, that has coefficient group $\Pi_{k p}(N)$, and that is carried by the $\left|\nabla^{k}(\cdot)\right|^{p}$ energy concentration set of the sequence.

Using these vague definitions, one has the vague general conjecture:

Relative to $\partial \mathbb{B}^{m}$, the boundary of the $u_{i}$ bubbled chain $B_{u_{i}}$ equals the $u$ topological singularity chain $S_{u}$.

The vagueness here concerns the precise definition of chain and boundary operation, and how one precisely obtains the bubbled chain $B_{u_{i}}$ from the sequence $u_{i}$ and the topological singular chain $S_{u}$ from $u$. From the cases we know, it is clear there is no single answer; it depends on the Sobolev space $W^{k, p}\left(\mathbb{B}^{m}, N\right)$, in particular the group $\Pi_{k p}(N)$.

In the special case $W^{1,2}\left(\mathbb{B}^{3}, \mathbb{S}^{2}\right)$, the relevant homotopy group is $\Pi_{2}\left(\mathbb{S}^{2}\right) \simeq \mathbb{Z}$, and [BBC] and GMS1] show that this 1 chain is precisely an integer-multiplicity 1 dimensional rectifiable current of finite mass (but possibly infinite boundary mass). The special case was essentially generalized to $k=1, N=\mathbb{S}^{p}$ in GMS2 and ABO. Here, the bubbled chain, now of dimension $m-p$, is again a rectifiable current.

The paper [HR1] treated $W^{1,3}\left(\mathbb{B}^{3}, \mathbb{S}^{2}\right)$. The relevant homotopy group is $\Pi_{3}\left(\mathbb{S}^{2}\right)$, which is again isomorphic to $\mathbb{Z}$. Here the bubbled 1 chain was shown to be possibly of infinite mass, and the notion of a "scan" was invented to describe precisely compactness and boundary properties. The paper [HR2] has able to handle bubbling in weak limits of smooth maps that corresponds to any nonzero homotopy 
class in the infinite nontorsion part of $\Pi_{p}(N)$. In this situation, the homotopy class of a map $w$ on the sphere $\mathbb{S}^{m-1}$ can again be described using a differential $m-1$ form $\Phi_{w}$ on $\mathbb{S}^{m-1}$. The form is derived by a special algebro-combinatoric construction (depending on the rational homotopy class) involving a family of $w$ pullbacks of forms on $N$ and their " $d^{-1}$ integrals". For example, in case $w: \mathbb{S}^{3} \rightarrow \mathbb{S}^{2}$, $\Phi_{w}=w^{\#} \omega_{\mathbb{S}^{2}} \wedge d^{*} \Delta^{-1} w^{\#} \omega_{\mathbb{S}^{2}}$. In general, this representation by a finite family of differential forms allows useful energy estimates involving certain Gauss integrals. For a weakly convergent sequence of smooth maps, the bubbled chain, which cannot usually be represented as a finite mass current, can be understood precisely as a rectifiable scan whose boundary is given by the topological singularities of the limit Sobolev map. Though we have a somewhat satisfactory description of bubbling and topological singularity in all nontorsion cases, the question of sequential weak density in these cases are still not resolved, even for the case $W^{1,3}\left(\mathbb{B}^{4}, \mathbb{S}^{2}\right)$.

Unfortunately representations of a homotopy class by differential forms are not available for torsion classes. In particular, if the relevant homotopy group of $N$ is completely torsion, then one requires other techniques to get energy estimates needed for questions about weak limits of smooth maps. The first such case is $W^{1,2}\left(\mathbb{B}^{3}, \mathbb{R} P^{2}\right)$, and was treated in $[\mathrm{PR}]$. Here $\Pi_{2}\left(\mathbb{R P}^{2}\right) \simeq \mathbb{Z}_{2}$, and, a main result, is that smooth maps are $W^{1,2}$ sequentially weakly dense because $p=2$.

\section{I.4 $W^{2,2}\left(\mathbb{B}^{5}, \mathbb{S}^{3}\right)$}

The present paper started with the modest goal of understanding analytic estimates for maps of $w: \mathbb{S}^{4} \rightarrow \mathbb{S}^{3}$ so as to understand weak convergence and sequential weak density for smooth maps from $\mathbb{B}^{5}$ to $\mathbb{S}^{3}$. Here, the appropriate homotopy group is $\Pi_{4}\left(\mathbb{S}^{3}\right)$, which is isomorphic to $\mathbb{Z}_{2}$. But geometric descriptions of this homotopy class of $v$ are not very simple. As discussed in Sections IV.2 and IV.3 below, they involve considering, for a smooth approximation $u$ of $v$, the total twisting of a $u$-pullback normal framing upon circulation around a generic fiber $u^{-1}\{y\}$. The twisting of the normal frame leads to an element of $\Pi_{1}(\mathbb{S O}(3)) \simeq \mathbb{Z}_{2}$. To analytically compute such a twisting involves integration of a derivative of a pull-back framing, hence a second derivative of the original map. So it is natural for this homotopy group to try to look for estimates in terms of the Hessian energy.

A representative of the single nonzero element in $\Pi_{4}\left(\mathbb{S}^{4}, \mathbb{S}^{3}\right)$ is the suspension of the Hopf map, $\mathbb{S H}: \mathbb{S}^{5} \rightarrow \mathbb{S}^{3}$, described explicitly in the next section. In Section II below, we slightly adapt [BPV], Th.5 by defining the subfamily

$$
\mathcal{R}=\left\{u \in \mathcal{R}_{0}\left(\mathbb{B}^{5}, \mathbb{S}^{3}\right): u \equiv \mathbb{S H}\left(\frac{x-a}{|x-a|}\right) \text { on } \mathbb{B}_{\delta_{0}}(a) \backslash\{a\} \text { for all } a \in \text { Sing } u \text { and some } \delta_{0}>0\right\}
$$

and then proving:

Lemma III.2 The family $\mathcal{R}$ is $W^{2,2}$ strongly dense in $W^{2,2}\left(\mathbb{B}^{5}, \mathbb{S}^{3}\right)$.

\section{I.5 Connection Length}

Given a finite subset $A$ of $\mathbb{B}^{5}$, one may define $\mathbb{Z}_{2}$ connection for $A$ (relative to $\partial \mathbb{B}^{5}$ ) as a finite disjoint union $\Gamma$ of finite length arcs embedded in $\overline{\mathbb{B}^{5}}$ whose union of endpoints is precisely $A \cup\left(\Gamma \cap \partial \mathbb{B}^{5}\right)$. Thus, each point of $A$ is joined by a unique arc in $\Gamma$ to either another point of $A$ or to a point of $\partial \mathbb{B}^{5}$.

It will simplify some constructions to use a minimal $\mathbb{Z}_{2}$ connection for $A$, that is, one having least length. It is not difficult to verify the existence and structure of a minimal $\mathbb{Z}_{2}$ connection for $A$. It simply consists of the disjoint union of finitely many closed intervals in $\mathbb{B}^{5}$ and finitely many radially pointing intervals having one endpoint in $\partial \mathbb{B}^{5}$. In $₫$ III.3. we show how individual maps in $\mathcal{R}$ can be weakly approximated by smooth maps by proving: 
Theorem III.1 (Singularity Cancellation) If $u \in \mathcal{R}, \Gamma$ is a minimal $\mathbb{Z}_{2}$ connection for Sing $v$, and $\varepsilon>0$, then there exists a smooth $u_{\varepsilon} \in W^{2,2}\left(\mathbb{B}^{5}, \mathbb{S}^{3}\right) \cap \mathcal{C}^{\infty}$ so that $u_{\varepsilon}(x)=u(x)$ whenever $\operatorname{dist}(x, \Gamma)>\varepsilon$ and

$$
\int_{\mathbb{B}^{5}}\left|\nabla^{2} u_{\varepsilon}\right|^{2} d x \leq \varepsilon+\int_{\mathbb{B}^{5}}\left|\nabla^{2} u\right|^{2} d x+c_{\mathbb{S H}} \mathcal{H}^{1}(\Gamma)
$$

where $c_{\mathbb{S H}}=\int_{\mathbb{S}^{4}}\left|\nabla_{\text {tan }}^{2}(\mathbb{S H})\right|^{2} d \mathcal{H}^{4}<\infty$.

See Remark III.1 concerning this constant.

The core of our work, however, involves proving:

Theorem IV.2 (Length Bound) For any $u \in \mathcal{R}$, Singu has a $\mathbb{Z}_{2}$ connection $\Gamma$ satisfying

$$
\mathcal{H}^{1}(\Gamma) \leq c \int_{\mathbb{B}^{5}}\left|\nabla^{2} u\right|^{2} d x
$$

for some absolute constant $c$.

Combining this length bound with Lemma II.2 and Theorem III.1 we readily establish, in Section V] that any Sobolev map in $W^{2,2}\left(\mathbb{B}^{5}, \mathbb{S}^{3}\right)$ has a $W^{2,2}$ weak approximation by a sequence of smooth maps.

We prove the length bound in Section IV by finding a suitable connection $\Gamma$ through three applications of the coarea formula. For a regular value $p \in \mathbb{S}^{3}$ for $u$, the fiber $\Sigma=u^{-1}\{p\}$ is a smooth surface with cone point singularities at Sing $u$. By the coarea formula, we may choose this $p$ so that

$$
\int_{u^{-1}\{p\}} \frac{|\nabla u|^{4}+\left|\nabla^{2} u\right|^{2}}{J_{3} u} d \mathcal{H}^{2} .
$$

Then we need to choose connectiong curves on $\Sigma$. To do this we choose an orthonormal frame $\tilde{\tau}_{1}, \tilde{\tau}_{2}, \tilde{\tau}_{3}$ of the normal bundle of the surface $\Sigma=u^{-1}\{p\}$ by ortho-normalizing the $v$ pull-backs of a basis of $\operatorname{Tan}\left(\mathbb{S}^{3}, p\right)$. Inequality (I.2) gives that

$$
\int_{\Sigma}\left|\nabla \tilde{\tau}_{j}\right| d \mathcal{H}^{2} \leq c \int_{\mathbb{B}^{5}}\left|\nabla^{2} u\right|^{2} d x .
$$

We show how a a.e. oriented 2 plane in $\mathbb{R}^{5}$ determines at every point $x \in \Sigma$, with a finite exceptional set $b_{1}, \ldots, b_{j}$, an orthogonal basis of $\operatorname{Nor}(\Sigma, x)$, thought of as a reference normal framing. There is a unique $\gamma(x) \in \mathbb{S O}(3) \simeq \mathbb{R P}^{3}$ and one gets some curves on $\Sigma$, with total length bounded by a multiple of $c \int_{\mathbb{B}^{5}}\left|\nabla^{2} u\right|^{2} d x$ by choosing $\gamma^{-1}(E)$ where $E$ is a suitable great $\mathbb{R P}^{2} \subset \mathbb{S O}(3)$. The curves starting at the some $a_{i}$ may end in either another $a_{k}$ or in $\partial \mathbb{B}^{5}$ or (unfortunately) in a point $b_{\ell}$ where the reference framing degenerates. More argument, including another use of the coarea formula is required in sections IV.8, IV.9 to find additional curves of controlled length connecting $b_{\ell}$ to another $b_{m}$ or to $\partial \mathbb{B}^{5}$. Putting all these curves together gives a $\mathbb{Z}_{2}$ connection for Sing $u$ satisfying the desired length bound.

\section{Preliminaries}

We will let $c$ denote an absolute constant whose value may change from statement to statement and which is usually easily estimable. Here for $0 \leq k \leq m$ and various $k$ dimensional subsets $A$ of $\mathbb{R}^{m}$,

$$
\int_{A} f d \mathcal{H}^{k}=\int_{A} f(y) d \mathcal{H}^{k} y
$$

will denote integration with respect to $k$ dimensional Hausdorff measure. However, in top dimension where $\mathcal{H}^{m}$ coincides with Lebesgue measure, we will use the use the standard notations $\int_{A} f d x=\int_{A} f(x) d x$. 
Lemma II.1 For each positive integer $m$, there is a positive constant $c_{m}$ so that

$$
\|v\|_{W^{2,2}\left(\mathbb{B}^{m}, N\right)}^{2} \leq c_{m}\left[(\operatorname{diam} N)^{2}+\int_{\mathbb{B}^{m}}\left|\nabla^{2} v\right|^{2} d x\right]
$$

for any compact Riemannian submanifold $N$ of $\mathbb{R}^{\ell}$ and $v \in W^{2,2}\left(\mathbb{B}^{m}, N\right)$.

Proof. Here $\|v\|_{W^{2,2}\left(\mathbb{B}^{m}, N\right)}^{2}=\int_{\mathbb{B}^{m}}\left(|v|^{2}+|\nabla v|^{2}+\left|\nabla^{2} v\right|^{2}\right) d x$. We clearly have the estimate

$$
\int_{\mathbb{B}^{m}}|v|^{2} d x \leq \mathcal{H}^{m}\left(\mathbb{B}^{m}\right)(\operatorname{diam} N)^{2}
$$

Moreover, by the Poincaré inequality,

$$
\begin{aligned}
\int_{\mathbb{B}^{m}}|\nabla v|^{2} d x & =\sum_{i=1}^{m} \int_{\mathbb{B}^{m}}\left|\frac{\partial v}{\partial x_{i}}\right|^{2} d x \leq \sum_{i=1}^{m} 2 \int_{\mathbb{B}^{m}}\left|\frac{\partial v}{\partial x_{i}}-\left(\frac{\partial v}{\partial x_{i}}\right)_{a v g}\right|^{2} d x+2 \int_{\mathbb{B}^{m}}\left|\left(\frac{\partial v}{\partial x_{i}}\right)_{a v g}\right|^{2} d x \\
& \leq 2 m \mathbf{C}_{\mathbb{B}^{m}} \int_{\mathbb{B}^{m}}\left|\nabla^{2} v\right|^{2} d x+\sum_{i=1}^{m} 2 \mathcal{H}^{m}\left(\mathbb{B}^{m}\right)\left|\left(\frac{\partial v}{\partial x_{i}}\right)_{a v g}\right|^{2}
\end{aligned}
$$

It only remains to bound $\left(\frac{\partial v}{\partial x_{i}}\right)_{a v g}$. We will do the case $i=1$, the cases $i \geq 2$ being similar. By Fubini's theorem and the absolutely continuity of $v$ on a.e. line in the $(1,0, \ldots, 0)$ direction,

$$
\begin{aligned}
& \mathcal{H}^{m}\left(\mathbb{B}^{m}\right)\left|\left(\frac{\partial v}{\partial x_{1}}\right)_{a v g}\right|=\left|\int_{\mathbb{B}^{m}} \frac{\partial v}{\partial x_{1}} d x\right|=\left|\int_{\mathbb{B}^{m-1}} \int_{-\sqrt{1-|y|^{2}}}^{\sqrt{1-|y|^{2}}} \frac{\partial v}{\partial x_{1}}\left(t, y_{1}, \ldots, y_{m-1}\right) d t d y\right| \\
& \leq \int_{\mathbb{B}^{m-1}}\left|v\left(\sqrt{1-|y|^{2}}, y_{1}, \ldots, y_{m-1}\right)-v\left(-\sqrt{1-|y|^{2}}, y_{1}, \ldots, y_{m-1}\right)\right| d y \leq \mathcal{H}^{m-1}\left(\mathbb{B}^{m-1}\right) \operatorname{diam} N
\end{aligned}
$$

\section{A Formula for the Suspension of the Hopf Map}

Let $\mathbb{H}: \mathbb{S}^{3} \rightarrow \mathbb{S}^{2}$ denote the standard Hopf map $[\mathrm{HR}]$ :

$$
\mathbb{H}\left(x_{1}, x_{2}, x_{3}, x_{4}\right)=\left(2 x_{1} x_{2}+2 x_{3} x_{4}, 2 x_{1} x_{4}-2 x_{2} x_{3}, x_{1}^{2}+x_{3}^{2}-x_{2}^{2}-x_{4}^{2}\right)
$$

and $\mathbb{S H I}: \mathbb{S}^{4} \rightarrow \mathbb{S}^{3}$ be its suspension:

$$
\operatorname{SH}\left(x_{0}, x_{1}, \cdots, x_{4}\right)=\left(x_{0}, \sqrt{1-x_{0}^{2}} \cdot \mathbb{H}\left(\frac{x_{1}}{\sqrt{x_{1}^{2}+\cdots+x_{4}^{2}}}, \ldots, \frac{x_{4}}{\sqrt{x_{1}^{2}+\cdots+x_{4}^{2}}}\right)\right) .
$$

The latter map generates the nonzero element of $\Pi_{4}\left(\mathbb{S}^{3}\right) \simeq \mathbb{Z}_{2}$. Also, its homogeneous degree 0 extension

$$
\mathbb{S H}(x /|x|) \in W^{2,2}\left(\mathbb{B}^{5}, \mathbb{S}^{3}\right)
$$

In particular, $\int_{\mathbb{B}^{5}}\left|\nabla^{2}(\mathbb{S H}(x /|x|))\right|^{2} d x=c_{\mathbb{S H}} \quad$ where

$$
c_{\mathbb{S H}}=\int_{\mathbb{S}^{4}}\left|\nabla_{\text {tan }}^{2}(\mathbb{S H H})\right|^{2} d \mathcal{H}^{4}<\infty
$$

While the explicit formula for a suspension of the Hopf map is handy for simplifying proofs, the constant $c_{\text {SHI }}$, which occurs in the conclusion of Theorem II.1 can, by Remark 11.1 be replaced by a more natural constant. 


\section{A Strongly Dense Family with Isolated Singularities}

Let $\mathcal{R}$ denote the class of $W^{2,2}\left(\mathbb{B}^{5}, \mathbb{S}^{3}\right)$ maps that are smooth except for finitely many suspension Hopf singularities. That is,

$$
\begin{aligned}
u \in \mathcal{R} & \Longleftrightarrow \\
u \in \mathcal{C}^{\infty}\left(\mathbb{B}^{5} \backslash\left\{a_{1}, \ldots, a_{m}\right\}, \mathbb{S}^{3}\right) \quad \text { and } \quad u(x) & =\mathbb{S H}\left(\frac{x-a_{i}}{\left|x-a_{i}\right|}\right) \quad \text { on } \quad \mathbb{B}_{\delta_{0}}\left(a_{i}\right) \backslash\left\{a_{i}\right\}
\end{aligned}
$$

for some finite subset $\left\{a_{1}, \ldots, a_{m}\right\}$ of $\mathbb{B}^{5}$ and some positive $\left.\delta_{0}<\min _{i}\left\{1-\left|a_{i}\right|, \min _{j \neq i}\left|a_{i}-a_{j}\right| / 2\right\}\right\}$.

\section{III.1 Strong Approximation by Maps in $\mathcal{R}$}

Lemma III.2 $\mathcal{R}$ is $W^{2,2}$ strongly dense in $W^{2,2}\left(\mathbb{B}^{5}, \mathbb{S}^{3}\right)$.

Proof. Theorem 5 of $[\mathrm{BPV}]$ gives the $W^{2,2}$ strongly density of the family $\mathcal{R}_{0}^{2,2}\left(\mathbb{B}^{5}, \mathbb{S}^{3}\right)$ of maps $v \in W^{2,2}\left(\mathbb{B}^{5}, \mathbb{S}^{3}\right)$ which are smooth except for a finite singular set $\left\{a_{1}, \ldots, a_{m}\right\}$ and which satisfy

$$
\limsup _{x \rightarrow a_{i}}\left(\left|x-a_{i}\right||\nabla v(x)|+\left|x-a_{i}\right|^{2}\left|\nabla^{2} v(x)\right|\right)<\infty,
$$

for $i=1, \ldots, m$. Thus, it suffices to show:

For each $v \in \mathcal{R}_{0}^{2,2}\left(\mathbb{B}^{5}, \mathbb{S}^{3}\right)$ and $\varepsilon>0$, there is a map $u \in \mathcal{R}$ so that $\|u-v\|_{W^{2,2}}^{2}<\varepsilon$.

Assuming Sing $v=\left\{a_{1}, \ldots, a_{m}\right\}$, we will obtain $u$ by modifying $v$ near each point $a_{i}$. First fix a positive $\eta<\frac{1}{2} \min \left\{\min _{i \neq j}\left|a_{i}-a_{j}\right|, \min _{k}\left(1-\left|a_{k}\right|\right)\right\}$ so that

$$
L=\max _{i} \sup _{0<\left|x-a_{i}\right|<\eta}\left(\left|x-a_{i}\right||\nabla v(x)|+\left|x-a_{i}\right|^{2}\left|\nabla^{2} v(x)\right|\right)<\infty
$$

We will proceed in two stages: First we will find a positive $\delta<\eta$ depending only on $L$ and then define a map $w \in W^{2,2}\left(\mathbb{B}^{5}, \mathbb{S}^{3}\right)$ so that

$$
w \equiv v \text { on } \mathbb{B}^{5} \backslash \cup_{i=1}^{m} \mathbb{B}_{\delta}\left(a_{i}\right),
$$

and, on each ball $\mathbb{B}_{\delta / 2}\left(a_{i}\right), w$ is degree-zero homogeneous about $a_{i}$, i.e.

$$
w(x)=w\left(a_{i}+\frac{x-a_{i}}{\left|x-a_{i}\right|}\right) \quad \text { for } \quad 0<\left|x-a_{i}\right|<\frac{1}{2} \delta .
$$

Second, we find a positive $\delta_{0}<<\frac{1}{2} \delta$, depending on $w \mid \cup_{i=1}^{m} \partial \mathbb{B}_{\delta / 2}\left(a_{i}\right)$, and a map $u \in \mathcal{R}$ with $u \equiv w$ on $\mathbb{B}^{5} \backslash \cup_{i=1}^{m} \mathbb{B}_{\delta_{0}}\left(a_{i}\right)$ and, on each ball $\mathbb{B}_{\delta_{0}}\left(a_{i}\right), u \equiv \mathbb{S H}\left(\frac{x-a_{i}}{\left|x-a_{i}\right|}\right)$.

For the first step, we first fix a smooth monotone increasing $\lambda:[0, \infty) \rightarrow\left[\frac{1}{2}, \infty\right)$ so that

$$
\lambda(t)= \begin{cases}1 / 2 & \text { for } \quad 0 \leq t \leq \frac{1}{2} \\ t & \text { for } t \geq 1\end{cases}
$$

Consider the unscaled situation of a map $V \in \mathcal{C}^{\infty}\left(\overline{\mathbb{B}^{5}} \backslash \mathbb{B}_{\frac{1}{2}}(0), \mathbb{S}^{3}\right)$ with $|\nabla V|+\left|\nabla^{2} V\right| \leq L$. Then we define the reparameterized map

$$
W(x)=V(\lambda(|x|) x),
$$

and see that, with respect to the radial variable $\rho=|x|$,

$$
\frac{\partial W}{\partial \rho} \equiv \frac{\partial V}{\partial \rho} \quad \text { on } \quad \partial \mathbb{B}^{5}, \quad \frac{\partial W}{\partial \rho} \equiv 0 \quad \text { on } \quad \overline{\mathbb{B}_{\frac{1}{2}}(0)} \backslash\{0\},
$$


Moreover, using explicit pointwise bounds for $\left|\lambda^{\prime}\right|$ and $\left|\lambda^{\prime \prime}\right|$, we readily find an explicit constant $C$ so that

$$
\begin{gathered}
|\nabla W(x)|+\left|\nabla^{2} W(x)\right| \leq C L \quad \text { for } \quad \frac{1}{2} \leq|x| \leq 1, \\
|x||\nabla W(x)|+|x|^{2}\left|\nabla^{2} W(x)\right| \leq C L \quad \text { for } \quad 0<|x|<\frac{1}{2} .
\end{gathered}
$$

Now we return to the original scale by defining $w$ to satisfy (III.6) and to have, for each point $x \in \mathbb{B}_{\delta}\left(a_{i}\right)$,

$$
w(x)=W\left(\frac{x-a_{i}}{\delta}\right) \text { where } V(x)=v\left(a_{i}+\delta x\right) .
$$

Then $w$ belongs to $W^{2,2}\left(\mathbb{B}^{5}, \mathbb{S}^{3}\right)$ and satisfies the estimate

$$
\max _{i} \sup _{0<\left|x-a_{i}\right|<\delta}\left(\left|x-a_{i}\right||\nabla w(x)|+\left|x-a_{i}\right|^{2}\left|\nabla^{2} w(x)\right|\right) \leq C L,
$$

hence,

$$
\begin{aligned}
\|w-v\|_{W^{2,2}}^{2} & =\sum_{i=1}^{m} \int_{\mathbb{B}_{\delta}\left(a_{i}\right)}\left(|w-v|^{2}+|\nabla w-\nabla v|^{2}+\left|\nabla^{2} w-\nabla^{2} v\right|^{2}\right) d x \\
& \leq 2 \sum_{i=1}^{m} \int_{\mathbb{B}_{\delta}\left(a_{i}\right)}\left(|w|^{2}+|v|^{2}+|\nabla w|^{2}+\left.\nabla v\right|^{2}+\left|\nabla^{2} w\right|^{2}+\left|\nabla^{2} v\right|^{2}\right) d x \leq c(1+L)^{2} \delta .
\end{aligned}
$$

So we easily choose $\delta$ so that $\|w-v\|_{W^{2,2}}^{2}<\frac{1}{4} \varepsilon$.

For the second step we note that any continuous homotopy between smooth maps of smooth manifolds can be made smooth; hence :

A smooth map $\phi: \mathbb{S}^{4} \rightarrow \mathbb{S}^{3}$ is smoothly homotopic $\begin{cases}\text { either to a constant } & \text { in case } \llbracket \phi \rrbracket=0 \in \Pi_{4}\left(\mathbb{S}^{4}, \mathbb{S}^{3}\right) \\ \text { or to } \llbracket \mathbb{S H} \rrbracket & \left.\text { in case } \llbracket \phi \rrbracket \neq 0 \in \Pi_{4}\left(\mathbb{S}^{4}, \mathbb{S}^{3}\right)\right) \text {. }\end{cases}$

For each $i=1, \ldots, m$, we apply this to the map $\phi_{i}(x)=w\left(a_{i}+\frac{1}{2} \delta x\right)$ to obtain a smooth homotopy $h_{i}:[0,1] \times \mathbb{S}^{4} \rightarrow \mathbb{S}^{3}$ which connects $\phi_{i}$ to $\mathbb{S H}$. Reparameterizing the time variable near 0 and 1 , we may assume

$$
h_{i}(t, y)= \begin{cases}\phi_{i}(y) & \text { for } t \text { near } 0 \\ (\mathbb{S H} H)(y) & \text { for } t \text { near } 1\end{cases}
$$

By smoothness, $K=\sup _{i}\left\|h_{i}\right\|_{W^{2,2}}<\infty$, and we will, for some $\delta_{0}<<\frac{1}{2} \delta$, define the map $u$ by

$$
\begin{gathered}
u \equiv w \text { on } \mathbb{B}^{5} \backslash \cup_{i=1}^{m} \mathbb{B}_{\delta_{0}}\left(a_{i}\right), \\
u(x)=h_{i}\left(2-2 \frac{\left|x-a_{i}\right|}{\delta_{0}}, \frac{x-a_{i}}{\delta_{0}}\right) \quad \text { for } \quad x \in \mathbb{B}_{\delta_{0}}\left(a_{i}\right) \backslash \mathbb{B}_{\frac{1}{2} \delta_{0}}\left(a_{i}\right), \\
u(x)=\mathbb{S H}\left(\frac{x-a_{i}}{\left|x-a_{i}\right|}\right) \quad \text { for } \quad x \in \mathbb{B}_{\frac{1}{2} \delta_{0}}\left(a_{i}\right) \backslash\{0\},
\end{gathered}
$$

for $i=1, \ldots, m$. One readily checks that $u \in \mathcal{R}$. Moreover, as in Step 1, we find that

$$
\|u-w\|_{W^{2,2}}^{2}=\sum_{i=1}^{m} \int_{\mathbb{B}_{\delta}\left(a_{i}\right)}\left(|u-w|^{2}+|\nabla u-\nabla w|^{2}+\left|\nabla^{2} u-\nabla^{2} w\right|^{2}\right) d x \leq c(1+K)^{4} \delta_{0} .
$$

So we easily choose $\delta_{0}$ small enough so that $\|u-w\|_{W^{2,2}}^{2}<\frac{1}{4} \varepsilon$ and obtain the desired estimate

$$
\|u-v\|_{W^{2,2}}^{2} \leq 2\|u-w\|_{W^{2,2}}^{2}+2\|w-v\|_{W^{2,2}}^{2}<\varepsilon .
$$




\section{III.2 Insertion of an $\mathbb{S H}$ Bubble into a Map from $\mathbb{B}^{4}$ to $\mathbb{S}^{3}$}

Arguing as in the proof of Lemma III.2 we first fix a monotone increasing smooth function $\mu$ on $[0, \infty)$ so that

$$
\mu(t)= \begin{cases}0 & \text { for } \quad 0 \leq t \leq \frac{1}{2} \\ t & \text { for } \quad t \geq 1\end{cases}
$$

$\left|\mu^{\prime}\right| \leq 3$, and $\left|\mu^{\prime \prime}\right| \leq 16$. We readily prove the following:

Lemma III.3 (Initial Reparameterization) There an absolute constant $C$ so that, for any smooth $f: \mathbb{B}^{4} \rightarrow \mathbb{S}^{3}$ and $0<\sigma<1$, the map

$$
f_{\sigma}: \mathbb{B}^{4} \rightarrow \mathbb{S}^{3}, \quad f_{\sigma}(y)=f\left(\mu\left(\sigma^{-1}|y|\right)|y|^{-1} \sigma y\right) \quad \text { for } y \in \mathbb{B}^{4},
$$

coincides with $f$ on $\mathbb{B}^{4} \backslash \mathbb{B}_{\sigma}^{4}$, is identically equal to $f(0)$ on $\mathbb{B}_{\sigma / 2}^{4}$, and satisfies

$$
\sup _{\mathbb{B}_{\sigma}^{4}}\left|\nabla f_{\sigma}\right| \leq C \sup _{\mathbb{B}_{\sigma}^{4}}\left|\nabla f_{\sigma}\right|, \quad \sup _{\mathbb{B}_{\sigma}^{4}}\left|\nabla^{2} f_{\sigma}\right| \leq C \sigma^{-1} \sup _{\mathbb{B}_{\sigma}^{4}}\left|\nabla^{2} f\right| .
$$

In particular,

$$
\int_{\mathbb{B}_{\sigma}^{4}}\left|\nabla^{2} f_{\sigma}\right|^{2} d x \leq C^{2} \mathcal{H}^{4}\left(\mathbb{B}^{4}\right) \sup _{\mathbb{B}_{\sigma}^{4}}\left|\nabla^{2} f\right|^{2} \sigma^{2}
$$

\section{Construction of an $\mathbb{S H}$ Bubble}

Recalling that $\mathbb{S H}(0,1,0,0,0)=(0,0,0,1)$, we will first slightly modify $\mathbb{S H}$ to be constant near $(0,1,0,0,1)$. Consider the spherical coordinate parameterization,

$$
\Upsilon:[0, \pi] \times \mathbb{S}^{3} \rightarrow \mathbb{S}^{4}, \quad \Upsilon(\rho, \omega)=\left((\sin \rho) \omega_{1}, \cos \rho,(\sin \rho) \omega_{2},(\sin \rho) \omega_{3},(\sin \rho) \omega_{4}\right) .
$$

Arguing again as in the proofs of Lemma \I.2 and Lemma 【I.3, we let

$$
M_{\rho_{0}}(\rho, \omega)=\left(\mu\left(\rho_{0}^{-1} \rho\right) \rho_{0} \rho, \omega\right) \quad \text { for } \quad 0 \leq \rho \leq \rho_{0}<<1 \quad \text { and } \omega \in \mathbb{S}^{3},
$$

and define $\Phi_{\rho_{0}}: \mathbb{S}^{4} \rightarrow \mathbb{S}^{4}$ by

$$
\Phi_{\rho_{0}}(y)= \begin{cases}\Upsilon \circ M_{\rho_{0}} \circ \Upsilon^{-1}\{y\} & \text { for } y \in \Upsilon\left(\left[0, \rho_{0}\right] \times \mathbb{S}^{4}\right) \\ \mathrm{y} & \text { otherwise }\end{cases}
$$

Then $\Phi_{\rho_{0}}$ is surjective, and maps the entire spherical cap

$$
\Omega_{\rho_{0} / 2}=\Upsilon\left(\left[0, \rho_{0} / 2\right) \times \mathbb{S}^{4}\right)=\mathbb{S}^{4} \cap \mathbb{B}_{2 \sin \left(\rho_{0} / 4\right)}^{5}((0,1,0,0,0))
$$

to its center point $(0,1,0,0,0)$.

We now consider the composition $\mathbb{S H} \circ \Phi_{\rho_{0}}$ The homotopy class is unchanged

$$
\llbracket \mathbb{S H} \circ \Phi_{\rho_{0}} \rrbracket=\llbracket \mathbb{S H} \rrbracket \neq 0 \in \Pi_{4}\left(\mathbb{S}^{3}\right) .
$$

Noting that $\rho_{0} \frac{\partial^{2} M_{\rho_{0}}}{\partial \rho^{2}}$ is bounded independent of $\rho_{0}$ and has support in $\Upsilon\left(\left[0, \rho_{0}\right] \times \mathbb{S}^{3}\right)$, we readily verify, as in (III.7), that

$$
\int_{\mathbb{S}^{4}}\left|\nabla_{\text {tan }}^{2}\left(\mathbb{S H} \circ \Phi_{\rho_{0}}\right)\right|^{2} d \mathcal{H}^{4}=\int_{\mathbb{S}^{4} \backslash \Omega_{\rho_{0}}}\left|\nabla_{\text {tan }}^{2}(\mathbb{S H})\right|^{2} d \mathcal{H}^{4}+\int_{\Omega_{\rho_{0}}}\left|\nabla_{\text {tan }}^{2}\left(\mathbb{S H} \circ \Phi_{\rho_{0}}\right)\right|^{2} d \mathcal{H}^{4} \rightarrow \quad c_{\mathbb{S H H}}+0
$$

as $\rho_{0} \rightarrow 0$. 
Since the stereographic projection

$$
\Pi: \mathbb{S}^{4} \backslash\{(0,1,0,0,0)\} \rightarrow \mathbb{R}^{4}, \quad \Pi\left(x_{0}, x_{1}, x_{2}, x_{3}, x_{4}\right)=\left(\frac{x_{0}}{1-x_{1}}, \frac{x_{2}}{1-x_{1}}, \frac{x_{3}}{1-x_{1}}, \frac{x_{4}}{1-x_{1}}\right)
$$

is conformal, we get the conformal diffeomorphism

$$
\Lambda_{\rho_{0}}: \overline{\mathbb{B}^{4}} \rightarrow \mathbb{S}^{4} \backslash \Omega_{\rho_{0} / 2}, \quad \Lambda_{\rho_{0}}(y)=\Pi^{-1}\left[\left(\frac{\sin \left(\rho_{0} / 2\right)}{1-\cos \left(\rho_{0} / 2\right)}\right) y\right]
$$

We see that

$$
\mathbb{S H} \circ \Phi_{\rho_{0}} \circ \Lambda_{\rho_{0}}: \overline{\mathbb{B}^{4}} \rightarrow \mathbb{S}^{3}
$$

is a smooth surjection which sends $\partial \mathbb{B}^{4}$ identically to $\mathbb{S H}(0,1,0,0,0)=(0,0,0,1)$.

We wish to have a similar map that has boundary values being a a possibly different constant $\xi \in \mathbb{S}^{3}$. To get a suitable formula, it will be handy to recall that $\mathbb{S}^{3}$, being identified with the unit quaternions,

$$
\left\{x_{1}+x_{2} \mathbf{i}+x_{3} \mathbf{j}+x_{4} \mathbf{k}: x_{1}^{2}+x_{2}^{2}+x_{3}^{2}+x_{4}^{2}=1\right\}
$$

has a product $\star$ and, in particular, that

$$
(0,0,0,-1) \star(0,0,0,1)=(-\mathbf{k}) \star \mathbf{k}=\mathbf{1}=(1,0,0,0) .
$$

So now we define, for every $\rho_{0}>0$ and $\xi \in \mathbb{S}^{3}$, the bubble

$$
\mathcal{B}_{\rho_{0}, \xi}: \mathbb{B}^{4} \rightarrow \mathbb{S}^{3}, \quad \mathcal{B}_{\rho_{0}, \xi} \equiv \xi \star(-\mathbf{k}) \star \mathbb{S H} \circ \Phi_{\rho_{0}} \circ \Lambda_{\rho_{0}}
$$

which is identically equal to $\xi$ on $\partial \mathbb{B}^{4}$. By the conformal invariance of the Hessian energy and (III.9),

$$
\int_{\mathbb{B}^{4}}\left|\nabla^{2} \mathcal{B}_{\rho_{0}, \xi}\right|^{2} d y=\int_{\mathbb{S}^{4}}\left|\nabla_{\text {tan }}^{2}\left(\mathbb{S H} \circ \Phi_{\rho_{0}}\right)\right|^{2} d \mathcal{H}^{4} \rightarrow \int_{\mathbb{S}^{4}}\left|\nabla_{\text {tan }}^{2}(\mathbb{S H H})\right|^{2} d \mathcal{H}^{4}=c_{\mathbb{S H}} \quad \text { as } \rho_{0} \rightarrow 0 .
$$

Now, for a smooth map $f: \mathbb{B}^{4} \rightarrow \mathbb{S}^{3}, 0<\sigma<1$, and $0<\rho_{0}<<1$, we define the "bubbled" map $f_{\sigma, \rho_{0}}: \mathbb{B}^{4} \rightarrow \mathbb{S}^{3}$

$$
f_{\sigma, \rho_{0}}(y)= \begin{cases}f_{\sigma}(y) & \text { for } \sigma / 2<|y|<1 \\ \mathcal{B}_{\rho_{0}, f(0)}(2 y / \sigma) & \text { for }|y| \leq \sigma / 2\end{cases}
$$

Note that $f_{\sigma, \rho_{0}}$ is continuous because both expressions equal $f(0)$ on $\partial \mathbb{B}_{\sigma / 2}^{4}$. Moreover, all the positive order derivatives of both expressions vanish here because the smooth map $f_{\sigma}$ is constant on $\mathbb{B}_{\sigma / 2}^{4}$ while the smooth reparameterization $\Phi_{\rho_{0}}$ is constant on $\Omega_{\rho_{0} / 2}$. So the map $f_{\sigma, \rho_{0}}$ is, in fact, smooth. Moreover, since the Hessian energy is invariant under change of scale,

$$
\begin{aligned}
\int_{\mathbb{B}^{4}}\left|\nabla^{2} f_{\sigma, \rho_{0}}\right|^{2} d y & =\int_{\mathbb{B}^{4} \backslash \mathbb{B}_{\sigma / 2}^{4}}\left|\nabla^{2} f_{\sigma}\right|^{2} d y+\int_{\mathbb{B}_{\sigma / 2}^{4}}\left|\nabla^{2}\left[\mathcal{B}_{\rho_{0}, f(0)}\left(\frac{2}{\sigma}(\cdot)\right)\right]\right|^{2} d y \\
& =\int_{\mathbb{B}^{4} \backslash \mathbb{B}_{\sigma}^{4}}\left|\nabla^{2} f\right|^{2} d y+\int_{\mathbb{B}_{\sigma}^{4} \backslash \mathbb{B}_{\sigma / 2}^{4}}\left|\nabla^{2} f_{\sigma}\right|^{2} d y+\int_{\mathbb{B}^{4}}\left|\nabla^{2} \mathcal{B}_{\rho_{0}, f(0)}\right|^{2} d y \\
& \rightarrow \int_{\mathbb{B}^{4}}\left|\nabla^{2} f\right|^{2} d y+0+\int_{\mathbb{B}^{4}}\left|\nabla^{2} \mathcal{B}_{\rho_{0}, f(0)}\right|^{2} d y \quad \text { as } \quad \sigma \rightarrow 0 .
\end{aligned}
$$

Note that the middle equation also gives, with (III.7) the bound

$$
\int_{\mathbb{B}^{4}}\left|\nabla^{2} f_{\sigma, \rho_{0}}\right|^{2} d y \leq\left(1+C^{2}\right) \sup _{\mathbb{B}^{4}}\left|\nabla^{2} f\right|^{2}+\int_{\mathbb{B}^{4}}\left|\nabla^{2} \mathcal{B}_{\rho_{0}, f(0)}\right|^{2} d y
$$

independent of $\sigma$. 


\section{III.3 Singularity Cancellation of a Map in $\mathcal{R}$}

Theorem III.1 If $u \in \mathcal{R}, \Gamma$ is a minimal $\mathbb{Z}_{2}$ connection for Sing $v$, and $\varepsilon>0$, then there exists a smooth $u_{\varepsilon} \in W^{2,2}\left(\mathbb{B}^{5}, \mathbb{S}^{3}\right) \cap \mathcal{C}^{\infty}$ so that $u_{\varepsilon}(x)=u(x)$ whenever $\operatorname{dist}(x, \Gamma)>\varepsilon$ and

$$
\int_{\mathbb{B}^{5}}\left|\nabla^{2} u_{\varepsilon}\right|^{2} d x \leq \varepsilon+\int_{\mathbb{B}^{5}}\left|\nabla^{2} u\right|^{2} d x+c_{\mathbb{S H}} \mathcal{H}^{1}(\Gamma)
$$

Proof. Note that, by slightly rescaling near $\partial \mathbb{B}^{5}$, we may assume that $u$ extends smoothly to a neighborhood of $\overline{\mathbb{B}^{5}}$.

First, using (III.11), we fix a positive $\rho_{0}$ to be small enough so that,

$$
\left.\left|c_{\mathrm{SH}}-\int_{\mathbb{B}^{4}}\right| \nabla^{2} \mathcal{B}_{\rho_{0}, \xi}\right|^{2} d y \mid<\frac{\varepsilon}{4\left(1+\mathcal{H}^{1}(\Gamma)\right)}
$$

for all $\xi \in \mathbb{S}^{3}$. Also we recall that a minimal $\mathbb{Z}_{2}$ connection for $u$ is the union of a finite family $\mathcal{I}$ of disjoint closed intervals $I=\left[a_{I}, b_{I}\right]$ where

$$
a_{I} \in \operatorname{Sing} u \quad \text { and } \quad b_{I} \in \begin{cases}\text { either } & \text { Sing } u \\ \text { or } & \partial \mathbb{B}^{5} \text { with } I \perp \partial \mathbb{B}^{5} .\end{cases}
$$

Second, we fix a positive $\delta_{0}$ so that:

(1) $\delta_{0}<\frac{1}{2} \min _{a \in \operatorname{Sing} u}\left\{1-|a|, \min _{a \neq \tilde{a} \in \operatorname{Sing} u}|a-\tilde{a}|\right\}$

(2) $\delta_{0}<\frac{1}{2} \min \{|x-\tilde{x}|: x \in I, \tilde{x} \in \tilde{I}, I \neq \tilde{I} \in \mathcal{I}\}$.

(3) $u \in \mathcal{C}^{\infty}\left(\mathbb{B}_{1+\delta_{0}}^{5} \backslash \operatorname{Sing} u, \mathbb{S}^{3}\right)$.

(4) $\delta_{0}<\left(1+c_{\mathbb{S H}}\right)^{-1}(1+\operatorname{card}(\operatorname{Sing} u))^{-1} \varepsilon / 5$

Our main step in constructing $u_{\varepsilon}$ will be to use, for each $I \in \mathcal{I}$, the bubble insertion of $₫ \amalg$ II.2 in each cross-section of a pinched cylindrical region $V_{I}$ of radius $\delta_{0} / 9$. Near the singular endpoints of $I, V_{I}$ is pinched to be a round cone with opening angle $2 \arctan \left(\frac{1}{9}\right)$.

To describe the explicit construction, we need some notation. With $I=\left[a_{I}, b_{I}\right] \in \mathcal{I}$ as above in (III.16), let

$$
|I|=\left|b_{I}-a_{I}\right|, \quad \mathbf{e}_{I}=\left(b_{I}-a_{I}\right) /|I| \in \mathbb{S}^{4}, \quad \Pi_{I}: \mathbb{R}^{5} \rightarrow \mathbb{R}, \quad \Pi_{I}(x)=x \cdot \mathbf{e}_{I}-a_{I} \cdot \mathbf{e}_{I},
$$

and $B_{I}(t, r)$ be the open ball in the 4 dimensional affine plane $\Pi_{I}^{-1}\{t\}$ with center $a_{I}+t \mathbf{e}_{I}$ and radius $r$. We now define $V_{I}$ to be the pinched cylindrical region

$$
V_{I}=\bigcup_{0<t<|I|} B_{I}\left(t, r_{I}(t)\right),
$$

by using a fixed smooth function $\nu:[0, \infty) \rightarrow[0,1]$ with

$$
\nu(t)= \begin{cases}t & \text { for } 0 \leq t \leq \frac{1}{2} \\ 1 & \text { for } 1 \leq t\end{cases}
$$

to define the smooth radius function

$$
r_{I}(t)= \begin{cases}\left(\delta_{0} / 9\right) t & \text { for } 0 \leq t \leq \frac{1}{2} \delta_{0} \\ \left(\delta_{0} / 9\right) \nu\left(t / \delta_{0}\right) & \text { for } \frac{1}{2} \delta_{0} \leq t \leq \delta_{0} \\ \delta_{0} / 9 & \text { for } \delta_{0} \leq t \leq|I|-\delta_{0} \\ \left(\delta_{0} / 9\right) \nu\left((|I|-t) / \delta_{0}\right) & \text { in case } b_{I} \in \operatorname{Sing} u \text { and }|I|-\delta_{0} \leq t \leq|I|-\frac{1}{2} \delta_{0} \\ \left(\delta_{0} / 9\right)(|I|-t) & \text { in case } b_{I} \in \operatorname{Sing} u \text { and }|I|-\frac{1}{2} \delta_{0} \leq t \leq|I| \\ \delta_{0} / 9 & \text { in case } b_{I} \in \partial \mathbb{B}^{5} \text { and }|I|-\delta_{0} \leq t \leq|I|\end{cases}
$$


Thus $\partial V_{I}$ is a smooth hypersurface except for the conepoint(s) $a_{I}$ (and $b_{I}$ in case $b_{I} \in \operatorname{Sing} u$ ).

For convenience, we fix an orthonormal basis $\mathbf{e}_{1}^{I}, \ldots, \mathbf{e}_{4}^{I}$ for the orthogonal complement of $\mathbb{R} \mathbf{e}_{I}$. For each $t \in \mathbb{R}$, we will use the affine similarity

$$
A_{I, t}: \mathbb{R}^{4} \rightarrow \mathbb{R}^{5}, \quad A_{I, t}\left(y_{1}, \ldots, y_{4}\right)=a_{I}+t \mathbf{e}_{I}+r_{I}(t)\left[y_{1} \mathbf{e}_{1}^{I}+\cdots+y_{4} \mathbf{e}_{4}^{I}\right]
$$

so that $A_{I, t}\left(\mathbb{B}^{4}\right)=B_{I}\left(t, r_{I}(t)\right)$.

For $0<\sigma<1$, we recall (III.12) and define the smooth reparameterized map

$$
v_{\sigma}(x)= \begin{cases}u(x) & \text { for } x \in \mathbb{B}^{5} \backslash \bigcup_{I \in \mathcal{I}} V_{I} \cup\left\{a_{I}\right\} \cup\left\{b_{I}\right\} \\ \left(u \circ A_{I, t}\right)_{\sigma, \rho_{0}}\left(A_{I, t}^{-1}(x)\right) & \text { for } x \in B_{I}\left(t, r_{I}(t)\right) .\end{cases}
$$

Observe that $v_{\sigma}$ actually coincides with $u(x)$ outside the " $\sigma$ thin" set $\bigcup_{I \in \mathcal{I}} V_{I}^{\sigma} \cup\left\{a_{I}\right\} \cup\left\{b_{I}\right\}$ where

$$
V_{I}^{\sigma}=\bigcup_{0<t<|I|} B_{I}\left(t, \sigma r_{I}(t)\right)
$$

The explicit formulas given above and in the earlier parts of $\$$ III.3 show the qualitative smoothness of $v_{\sigma}$ on $\mathbb{B}^{5} \backslash$ Sing $u$. Our next goal is to verify that

$$
\limsup _{\sigma \rightarrow 0} \sum_{I \in \mathcal{I}} \int_{V_{I}^{\sigma}}\left|\nabla^{2} v_{\sigma}\right|^{2} d x<c_{\mathbb{S H}} \mathcal{H}(\Gamma)+\frac{\varepsilon}{2} .
$$

We define

$$
J_{I}=\left\{t: \frac{1}{2} \delta_{0} \leq t \leq\left\{\begin{array}{ll}
|I|-\frac{1}{2} \delta_{0} & \text { in case } b_{I} \in \operatorname{Sing} u \\
|I| & \text { in case } b_{I} \in \partial \mathbb{B}^{5}
\end{array}\right\}\right.
$$

and note that on $J_{I}$, the scaling factor $r_{I}(t)$ satisfies $\frac{1}{18} \delta \leq r_{I}(t) \leq \frac{1}{9} \delta_{0}$ while $\left|r_{I}^{\prime}(t)\right|$ and $\left|r_{I}^{\prime \prime}(t)\right|$ are bounded. We will use the corresponding truncated sets

$$
W_{I} \equiv V_{I} \cap \Pi_{I}^{-1}\left(J_{I}\right)=\bigcup_{t \in J_{I}} B_{I}\left(t, r_{I}(t)\right), \quad W_{I}^{\sigma} \equiv V_{I}^{\sigma} \cap \Pi_{I}^{-1}\left(J_{I}\right)=\bigcup_{t \in J_{I}} B_{I}\left(t, \sigma r_{I}(t)\right) .
$$

We have the pointwise bound

$$
L=\sup _{W_{I}}\left(|\nabla u|^{2}+\left|\nabla^{2} u\right|^{2}\right)<\infty
$$

hence, by (III.14),

$$
\sup _{t \in J_{I}} \sup _{0<\sigma<1} \int_{\mathbb{B}^{4}}\left|\nabla^{2}\left(u \circ A_{I, t}\right)_{\sigma, \rho_{0}}\right|^{2} d y<\infty .
$$

Note that the orthogonality of the five vectors $\mathbf{e}_{I}, \mathbf{e}_{1}^{I}, \cdots, \mathbf{e}_{4}^{I}$ lead to the decomposition of the squared Hessian norm into pure second partial derivatives

$$
\left|\nabla^{2}(\cdot)\right|^{2}=\left|\nabla_{\mathbf{e}_{I}, \mathbf{e}_{I}}(\cdot)\right|^{2}+\mid\left(\left.\nabla_{\mathbf{e}_{1}^{I}, \mathbf{e}_{1}^{I}(\cdot)}\right|^{2}+\cdots+\mid\left(\left.\nabla_{\mathbf{e}_{4}^{I}, \mathbf{e}_{4}^{I}}(\cdot)\right|^{2},\right.\right.
$$

which we will abbreviate as $\left|\nabla_{\mathbf{e}_{I}}^{2}(\cdot)\right|^{2}+\left|\nabla_{\mathbf{e}_{I}^{\perp}}^{2}(\cdot)\right|^{2}$.

It follows from Fubini's Theorem, the conformal invariance of the 4 dimensional Hessian energy, (III.13), dominated convergence, and (III.15) that

$$
\begin{aligned}
\int_{W_{I}^{\sigma}}\left|\nabla_{\mathbf{e}_{I}^{\perp}}^{2} v_{\sigma}\right|^{2} d x & =\int_{t \in J_{I}} \int_{B_{I}\left(t, r_{I}(t)\right)}\left|\nabla_{\mathbf{e}_{I}^{\perp}}^{2} v_{\sigma}\right|^{2} d \mathcal{H}^{4} d t \\
& =\int_{t \in J_{I}} \int_{\mathbb{B}^{4}}\left|\nabla^{2}\left(u \circ A_{I, t}\right)_{\sigma, \rho_{0}}\right|^{2} d y d t \\
& \rightarrow \int_{t \in J_{I}}\left[0+\int_{\mathbb{B}^{4}}\left|\nabla^{2} \mathcal{B}_{\rho_{0}, u\left(a_{I}+t \mathbf{e}_{I}\right)}\right|^{2} d y\right] d t \leq c_{\mathbb{S H}}|I|+\frac{\varepsilon|I|}{4\left(1+\mathcal{H}^{1}(\Gamma)\right)},
\end{aligned}
$$


as $\sigma \rightarrow 0$. Thus

$$
\limsup _{\sigma \rightarrow 0} \sum_{I \in \mathcal{I}} \int_{W_{I}^{\sigma}}\left|\nabla_{\mathbf{e}_{I}^{\perp}}^{2} v_{\sigma}\right|^{2} d x \leq \sum_{I \in \mathcal{I}}\left[c_{\mathbb{S H}}|I|+\frac{\varepsilon|I|}{4\left(1+\mathcal{H}^{1}(\Gamma)\right)}\right] \leq c_{\mathbb{S H H}} \mathcal{H}^{1}(\Gamma)+\varepsilon / 4 .
$$

To get the full squared Hessian integral $\int_{W_{I}^{\sigma}}\left|\nabla^{2} v_{\sigma}\right|^{2} d x$, we also need to consider $\int_{W_{I}^{\sigma}}\left|\nabla_{\mathbf{e}_{I}}^{2} v_{\sigma}\right|^{2} d x$, which involves computing $\frac{\partial^{2}}{\partial t^{2}}$ of various terms. To estimate the last integral, it again suffices by Fubini's theorem, Lemma ПI.3. (II.12), and changing variables to consider

$$
\int_{t \in J_{I}}\left[\int_{\mathbb{B}_{\sigma}^{4} \backslash \mathbb{B}_{\sigma / 2}^{4}} \mid \frac{\partial^{2}}{\partial t^{2}}\left(\left.u \circ A_{I, t)}\right|^{2} d y d t+\int_{\mathbb{B}_{\sigma / 2}^{4}}\left|\frac{\partial^{2}}{\partial t^{2}}\left[\mathcal{B}_{\rho_{0}, u\left(a_{I}+t \mathbf{e}_{I}\right)}\left(\frac{2}{\sigma}(\cdot)\right)\right]\right|^{2} d y\right] d t .\right.
$$

The chain rule and the bounds of $\left|r^{\prime}\right|$ and $\left|r^{\prime \prime}\right|$ on $J_{I}$ give the pointwise bound

$$
\left|\frac{\partial^{2}}{\partial t^{2}}\left(u \circ A_{I, t}\right)\right|=\left|\frac{\partial^{2}}{\partial t^{2}}\left[u\left(a_{I}+t \mathbf{e}_{I}+r_{I}(t) y\right)\right]\right| \leq c L,
$$

and definition (III.10) gives the bound

$$
\left|\frac{\partial^{2}}{\partial t^{2}} \mathcal{B}_{\rho_{0}, u\left(a_{I}+t \mathbf{e}_{I}\right)}\right|=\left|\frac{\partial^{2}}{\partial t^{2}}\left[u\left(a_{I}+t \mathbf{e}_{I}\right) \star(-\mathbf{k}) \star \mathbb{S H} \circ \Phi_{\rho_{0}} \circ \Lambda_{\rho_{0}}\right]\right| \leq L .
$$

Integrating implies that (III.21) is bounded by $(c+1) L|I| \mathcal{H}^{4}\left(\mathbb{B}_{\sigma}^{4}\right)$, and we deduce that

$$
\lim _{\sigma \rightarrow 0} \int_{W_{I}^{\sigma}}\left|\nabla_{\mathbf{e}_{I}}^{2} v_{\sigma}\right|^{2} d x=0
$$

Next we consider the conical end(s) $V_{I}^{\sigma} \backslash W_{I}^{\sigma}$. By our choice of $\delta_{0}, u$ is degree-0 homogeneous about $a_{I}$ on the region $\mathbb{B}_{\delta_{0}}^{5}\left(a_{I}\right)$. It follows that all of the normalized bubbled functions $\left(u \circ A_{I, t}\right)_{\sigma, \rho_{0}}$ coincide for $0<t \leq \sigma / 2$. Thus, in the one conical end $V_{I}^{\sigma} \cap \Pi_{I}^{-1}\left(0, \delta_{0} / 2\right], v_{\sigma}$ is also degree- 0 homogeneous about $a_{I}$. So we can easily estimate the Hessian integral there by using spherical coordinates about $a_{I}$. Note that radial projection of the 4 dimensional Euclidean ball $V_{I}^{\sigma} \cap \Pi^{-1}\left\{\delta_{0} / 2\right\}$ onto the small spherical cap $V_{I}^{\sigma} \cap \partial \mathbb{B}_{\delta_{0} / 2}^{5}$ is a smooth diffeomorphism with easily computed $\mathcal{C}^{2}$ bounds on it and its inverse. In particular, we see that, for $\sigma$ sufficiently small,

$$
E_{\sigma} \equiv \int_{V_{I}^{\sigma} \cap \partial \mathbb{B}_{\delta_{0} / 2}^{5}\left(a_{I}\right)}\left|\nabla_{t a n}^{2} v_{\sigma}\right|^{2} d \mathcal{H}^{4} \leq 2 \int_{\mathbb{B}_{\sigma}^{4}}\left|\nabla^{2}\left(u \circ A_{I, \delta_{0} / 2}\right)_{\sigma, \rho_{0}}\right|^{2} d y<2+2 c_{\mathbb{S H}} .
$$

By our initial choice (4) of $\delta_{0}$ we find that, for such $\sigma$,

$$
\int_{V_{I}^{\sigma} \cap \Pi_{I}^{-1}\left(0, \delta_{0} / 2\right]}\left|\nabla^{2} v_{\sigma}\right|^{2} d x \leq\left(\delta_{0} / 2\right) E_{\sigma} \leq(1+\operatorname{card}(\operatorname{Sing} u))^{-1} \varepsilon / 5 .
$$

In case $b_{I} \in \operatorname{Sing} u$, we make a similar estimate near $b_{I}$. In any case, we now have

$$
\limsup _{\sigma \rightarrow 0} \sum_{I \in \mathcal{I}} \int_{V_{I}^{\sigma} \backslash W_{I}^{\sigma}}\left|\nabla^{2} v_{\sigma}\right|^{2} d x \leq \varepsilon / 5
$$

which together with (III.19) and (III.22), gives the desired Hessian integral estimate (III.17) for $v_{\sigma}$.

Now using (III.17), we are ready to fix a positive $\sigma_{0}<1$ so that

$$
\sum_{I \in \mathcal{I}} \int_{V_{I}^{\sigma_{0}}}\left|\nabla^{2} v_{\sigma_{0}}\right|^{2} d x \leq c_{\mathbb{S H}} \mathcal{H}^{1}(\Gamma)+\varepsilon / 2
$$


The final step will be to modify $v_{\sigma_{0}}$ to get $u_{\varepsilon}$. The map $v_{\sigma_{0}}$ is smooth on $\overline{\mathbb{B}^{5}} \backslash$ Sing $u$ and is degree-0 homogeneous about each point $a \in \operatorname{Sing} u$, in the ball $\mathbb{B}_{\delta_{0} / 2}^{5}(a)$.

For each such $a$, consider the normalized map given by rescaling $v_{\sigma_{0}} \mid \partial \mathbb{B}_{\delta_{0} / 2}^{5}(a)$, namely,

$$
g_{a}: \mathbb{S}^{4} \rightarrow \mathbb{S}^{3}, \quad g_{a}(x)=v_{\sigma_{0}}\left[a+\left(\delta_{0} / 2\right) x\right]
$$

We claim that, in $\Pi_{4}\left(\mathbb{S}^{3}\right) \simeq \mathbb{Z}_{2}$, the homotopy class $\llbracket g_{a} \rrbracket$ is zero. To see this, suppose that $a=a_{I}$ and first note that the restriction of the original map $u \mid \partial \mathbb{B}_{\delta_{0} / 2}^{5}(a)$ gives the nonzero class $\llbracket \mathbb{S H} \rrbracket \in \Pi_{4}\left(\mathbb{S}^{3}\right)$ by the definition of $\mathcal{R}$. Second, we slightly reparameterized $u \mid \partial \mathbb{B}_{\delta_{0} / 2}^{5}(a)$ near the point $a+\left(\delta_{0} / 2\right) \mathbf{e}_{I}$ to have constant value $\xi_{a_{I}}=(\mathbb{S H H})\left(\mathbf{e}_{I}\right)$ in a small spherical cap of radius $\sigma_{0} \delta_{0} / 2$. The resulting reparameterized map $\tilde{u}_{a}$ still induces the nonzero homotopy class in $\Pi_{4}\left(\mathbb{S}^{3}\right)$. Third, in forming the map $v_{\sigma} \mid \partial \mathbb{B}_{\delta_{0} / 2}^{5}(a)$, we inserted a bubble in the small cap of constancy of $\tilde{u}_{a}$. This insertion gives the resulting sum in $\Pi_{4}\left(\mathbb{S}^{3}\right)$ :

$$
\llbracket g_{a} \rrbracket=\llbracket \tilde{u}_{a} \rrbracket+\llbracket \xi_{a_{I}} \star \mathbb{S H} \circ \Phi_{\rho_{0}} \rrbracket=\llbracket \mathbb{S H} \rrbracket+\llbracket \mathbb{S H} \circ \Phi_{\rho_{0}} \rrbracket=2 \llbracket \mathbb{S H} \rrbracket=0
$$

by (III.8). The same is true in case $a$ is a second endpoint $b_{I}$.

Now, as in the proof of Lemma III.2 $g_{a}$ is homotopic to a constant, and we may we may fix a smooth homotopy $h_{a}:[0,1] \times \mathbb{S}^{4} \rightarrow \mathbb{S}^{3}$ so that

$$
h_{a}(t, y)= \begin{cases}g_{a}(y) & \text { for } t \text { near } 0 \\ (1,0,0,0) & \text { for } t \text { near } 1 \\ . & \end{cases}
$$

Thus the map

$$
H_{a}: \overline{\mathbb{B}^{5}} \rightarrow \mathbb{S}^{3}, \quad H_{a}(x)=h_{a}(1-|x|, x /|x|) \text { for } 0<|x| \leq 1, \quad H_{a}(0)=(1,0,0,0),
$$

is smooth. Moreover, for $0<\tau \leq \delta_{0} / 2$,

$$
w_{\tau}: \bigcup_{a \in \operatorname{Sing} u} \mathbb{B}_{\tau}^{5}(a) \rightarrow \mathbb{S}^{3}, \quad w_{\tau}(x)=H_{a}\left(\frac{x-a}{\tau}\right) \text { for } x \in \mathbb{B}_{\tau}^{5}(a),
$$

satisfies

$$
\int_{\mathbb{B}_{\tau}^{5}(a)}\left|\nabla^{2} w_{\tau}\right|^{2} d x=\tau \int_{\mathbb{B}^{5}}\left|\nabla H_{a}\right|^{2} d x
$$

and we can fix a positive $\tau_{0} \leq \delta_{0} / 2$ so that

$$
\sum_{a \in \operatorname{Sing} u} \int_{\mathbb{B}_{\tau_{0}}^{5}(a)}\left|\nabla^{2} w_{\tau_{0}}\right|^{2} d x<\varepsilon / 2 .
$$

Finally we define the desired map $u_{\varepsilon}: \mathbb{B}^{5} \rightarrow \mathbb{S}^{3}$ by :

$$
u_{\varepsilon}(x)= \begin{cases}v_{\sigma_{0}}(x) & \text { for } x \in \cup_{I \in \mathcal{I}} V_{I}^{\sigma_{0}} \backslash \cup_{a \in \operatorname{Sing} u} \mathbb{B}_{\tau_{0}}^{5}(a) \\ w_{\tau_{0}}(x) & \text { for } x \in \cup_{a \in \operatorname{Sing} u} \mathbb{B}_{\tau_{0}}^{5}(a) \\ u(x) & \text { otherwise. }\end{cases}
$$

We easily verify that $u_{\varepsilon}$ is smooth and coincides with $u$ outside an $\varepsilon$ neighborhood of $\Gamma$ because $\sigma_{0} \delta_{0} / 9<\varepsilon$ and $\tau_{0} \leq \delta_{0} / 2<\varepsilon$. Moreover, by (III.23) and (III.24),

$$
\begin{aligned}
\int_{\mathbb{B}^{5}}\left|\nabla^{2} u_{\varepsilon}\right|^{2} d x & \leq \sum_{I \in \mathcal{I}} \int_{V_{I}^{\sigma_{0}}}\left|\nabla^{2} v_{\sigma_{0}}\right|^{2} d x+\sum_{a \in \operatorname{Sing} u} \int_{\mathbb{B}_{\tau_{0}}^{5}(a)}\left|\nabla^{2} w_{\tau_{0}}\right|^{2} d x+\int_{\mathbb{B}^{5}}\left|\nabla^{2} u\right|^{2} d x \\
& \leq c_{\mathbb{S H}} \mathcal{H}^{1}(\Gamma)+\frac{\varepsilon}{2}+\frac{\varepsilon}{2}+\int_{\mathbb{B}^{5}}\left|\nabla^{2} u\right|^{2} d x .
\end{aligned}
$$


Corollary III.1 If $u$ and $u_{\varepsilon}$ are as in Theorem [II.1, then $u_{\varepsilon}$ approaches $u, W^{2,2}$ weakly as $\varepsilon \rightarrow 0$.

Proof. One has the strong $L^{2}$ convergence $\lim _{\varepsilon \rightarrow 0}\left\|u_{\varepsilon}-u\right\|_{L^{2}}=0$, because the $u_{\varepsilon}$ are uniformly bounded (by 1) and approach $u$ pointwise on $\mathbb{B}^{5} \backslash \operatorname{Sing} u$. Moreover, for any sequence $1 \geq \varepsilon_{i} \downarrow 0$, we have by Theorem II.1 and Lemma II.1, the bound

$$
\sup _{i}\left\|u_{\varepsilon_{i}}\right\|_{W^{2,2}}^{2}<c_{m}\left(4+\int_{\mathbb{B}^{5}}\left|\nabla^{2} u\right|^{2} d x+c_{\mathbb{S} \mathbb{H}} \mathcal{H}^{1}(\Gamma)\right)<\infty .
$$

By the weak* (=weak) compactness of the closed ball in $W^{2,2}\left(\mathbb{B}^{5}, \mathbb{R}^{\ell}\right)$, the sequence $u_{\varepsilon_{i}}$ contains a subequence $u_{\varepsilon_{i^{\prime}}}$ that is $W^{2,2}$ weakly convergent to some $w \in W^{2,2}\left(\mathbb{B}^{5}, \mathbb{R}^{\ell}\right)$. But, $w$, being by Rellich's theorem, the strong $L^{2}$ limit of the $u_{\varepsilon_{i^{\prime}}}$, must necessarily be the original map $u$. Since any subsequence of $u_{\varepsilon}$ subconverges to the same limit $u$ and since the weak* (=weak) $W^{2,2}$ topology on bounded sets is metrizable, the original family $u_{\varepsilon}$ converges $W^{2,2}$ weakly to $u$.

Remark III.1 In Theorem III.1, one may replace $c_{\mathrm{SH}}$ by the optimal constant

$$
\tilde{c}_{\mathbb{S H}}=\inf \left\{\int_{\mathbb{S}^{4}}\left|\nabla_{t a n}^{2} \omega\right|^{2} d \mathcal{H}^{4}: \omega \in \mathcal{C}^{\infty}\left(\mathbb{S}^{4}, \mathbb{S}^{3}\right) \text { and } \llbracket \omega \rrbracket=\llbracket \mathbb{S H} \rrbracket\right\} \text {. }
$$

Here, for any $\omega$ as above, we can first $W^{2,2}$ strongly approximate $u$ by a map which equals $\omega(x-a) /|x-a|$ in $\mathbb{B}_{\delta_{1}}(a)$ for all $a \in \operatorname{Sing} u$ and some $0<\delta_{1}<<\delta_{0}$. Then we repeat the proofs with $\mathbb{S H}$ replaced by $\omega$.

\section{Connecting Singularities with Controlled Length}

Suppose $u \in \mathcal{R}$ with $\operatorname{Sing} u=\left\{a_{1}, a_{2}, \ldots, a_{m}\right\}$ as above. Our goal in this section is to connect the singular points $a_{i}$, together in pairs or to $\partial \mathbb{B}^{5}$, by some union of curves whose total length is bounded by an absolute constant multiple of the Hessian energy, that is,

$$
c \int_{\mathbb{B}^{5}}\left|\nabla^{2} u\right|^{2} d x
$$

This is therefore a bound on the length of a minimal connection for Sing $u$, which will allow us, in Theorem V.3 below, to combine Lemma III.2 and Theorem III.1 to obtain the desired sequential weak density of $\mathcal{C}^{\infty}\left(\mathbb{B}^{5}, \mathbb{S}^{3}\right)$ in $W^{2,2}\left(\mathbb{B}^{5}, \mathbb{S}^{3}\right)$.

Using the surjectivity of the suspension of the Hopf map, we readily verify that each regular value $p \in \mathbb{S}^{3} \backslash\{(-1,0,0,0),(1,0,0,0)\}$ of $u$ gives a level surface

$$
\Sigma=u^{-1}\{p\}
$$

which necessarily contains all the singular points $a_{i}$ of $u$. Note that $\Sigma=u^{-1}\{p\}$ is smoothly embedded away from the $a_{i}$ with standard orientation $\omega_{\Sigma} \equiv * u^{\#} \omega_{\mathbb{S}^{3}} /\left|u^{\#} \omega_{\mathbb{S}^{3}}\right|$, induced from $u$. Concerning the behavior near $a_{i}$, the punctured neighborhood

$$
\Sigma \cap \mathbb{B}_{\delta_{0}}\left(a_{i}\right) \backslash\left\{a_{i}\right\}
$$

is simply a truncated cone whose boundary

$$
\Gamma_{i}=\Sigma \cap \partial \mathbb{B}_{\delta_{0}}\left(a_{i}\right)
$$

is a planar circle in the 3 -sphere $\partial \mathbb{B}_{\delta_{0}}\left(a_{i}\right) \cap\left(\left\{\delta p_{0}\right\} \times \mathbb{R}^{4}\right)$ where $p=\left(p_{0}, p_{1}, p_{2}, p_{3}\right)$.

We will eventually choose the desired "connecting" curves all to lie on one such level surface $\Sigma$. 


\section{IV.1 Estimates for Choosing the Level Surface $\Sigma=u^{-1}\{p\}$}

We first recall the 3 Jacobian $J_{3} u=\left\|\wedge_{3} D u\right\|$ and apply the coarea formula [Fe, $\S 3.2 .12$ with

$$
g=\frac{|\nabla u|^{4}+\left|\nabla^{2} u\right|^{2}}{J_{3} u}
$$

to obtain the relation

$$
\int_{\mathbb{S}^{3}} \int_{u^{-1}\{p\}} \frac{|\nabla u|^{4}+\left|\nabla^{2} u\right|^{2}}{J_{3} u} d \mathcal{H}^{2} d \mathcal{H}^{3} p=\int_{\mathbb{B}^{5}}\left(|\nabla u|^{4}+\left|\nabla^{2} u\right|^{2}\right) d x .
$$

Moreover, since $\|u\|_{L^{\infty}}=1$, we also have (see $\underline{\mathrm{MR}}$ ) the integral inequality

$$
\int_{\mathbb{B}^{5}}|\nabla u|^{4} \leq c \int_{\mathbb{B}^{5}}\left|\nabla^{2} u\right|^{2} d x
$$

In case $u$ is constant on $\partial \mathbb{B}^{5}$, we may verify this by computing

$$
\begin{aligned}
\int_{\mathbb{B}^{5}}|\nabla u|^{4} & =\int_{\mathbb{B}^{5}}(\nabla u \cdot \nabla u)|\nabla u|^{2} d x \\
& =\int_{\mathbb{B}^{5}}\left[\operatorname{div}\left(u \nabla u|\nabla u|^{2}\right)-u \cdot \Delta u|\nabla u|^{2}-u \nabla u \cdot \nabla\left(|\nabla u|^{2}\right)\right] d x \\
& \leq 0+5 \int_{\mathbb{B}^{5}}\left|\nabla^{2} u\right||\nabla u|^{2} d x+2 \int_{\mathbb{B}^{5}}\left|\nabla^{2} u\right||\nabla u|^{2} d x \\
& \leq \frac{1}{2} \int_{\mathbb{B}^{5}}|\nabla u|^{4} d x+\frac{49}{2} \int_{\mathbb{B}^{5}}\left|\nabla^{2} u\right|^{2} d x .
\end{aligned}
$$

In the general case, we write $u=\sum_{i=1}^{\infty} \lambda_{i} u$ where $\left\{\lambda_{i}\right\}$ is a partition of unity adapted to a family of Whitney cubes for $\mathbb{B}^{5}$. See $\mathrm{MR}$. (The above inequality is true even with the constraint $\|u\|_{B M O} \leq 1$ in place of $\|u\|_{L^{\infty}} \leq 1$ [MR.)

By (IV.25) and (IV.26) we may now choose a regular value $p \in \mathbb{S}^{3}$ of $u$ so that

$$
\int_{u^{-1}\{p\}} \frac{|\nabla u|^{4}+\left|\nabla^{2} u\right|^{2}}{J_{3} u} d \mathcal{H}^{2} \leq c \int_{\mathbb{B}^{5}}\left|\nabla^{2} u\right|^{2} d x .
$$

By increasing $c$ we will also insist that $\left|p_{0}\right|$ is small, say, $\left|p_{0}\right|<1 / 100$. This smallness will be useful in guaranteeing that each tangent plane Tan $(\Sigma, x)$, for $x \in \Sigma \cap \cup_{i=1}^{m} \mathbb{B}_{\delta_{0}}\left(a_{i}\right) \backslash\left\{a_{i}\right\}$, is close to $\{0\} \times \mathbb{R}^{4}$.

\section{IV.2 A Pull-back Normal Framing for $\Sigma=u^{-1}\{p\}$}

Suppose again that $p=\left(p_{0}, p_{1}, p_{2}, p_{3}\right) \in \mathbb{S}^{3} \backslash\{(-1,0,0,0),(1,0,0,0)\}$ is a regular value of $u$. Then

$$
\eta_{1}=\left(-\sqrt{1-p_{0}^{2}}, \frac{p_{0} p_{1}}{\sqrt{1-p_{0}^{2}}}, \frac{p_{0} p_{2}}{\sqrt{1-p_{0}^{2}}}, \frac{p_{0} p_{3}}{\sqrt{1-p_{0}^{2}}}\right)
$$

is the unit vector tangent at $p$ to the geodesic that runs from $(1,0,0,0)$ through $p$ to $(-1,0,0,0)$. We may choose two other vectors

$$
\eta_{2}, \eta_{3} \in \operatorname{Tan}\left(\left\{p_{0}\right\} \times \sqrt{1-p_{0}^{2}} \mathbb{S}^{2}, p\right) \subset \operatorname{Tan}\left(\mathbb{S}^{3}, p\right)
$$


so that $\eta_{1}, \eta_{2}, \eta_{3}$ becomes an orthonormal basis for $\operatorname{Tan}\left(\mathbb{S}^{3}, p\right)$. Since $p$ is a regular value for $u$, these three vectors lift to three unique smooth linearly independent normal vectorfields $\tau_{1}, \tau_{2}, \tau_{3}$ along $\Sigma=u^{-1}\{p\}$. That is, at each point $x \in \Sigma$,

$$
\tau_{j}(x) \perp \Sigma \text { at } x \text { and } D u(x)\left[\tau_{j}(x)\right]=\eta_{j}
$$

for $j=1,2,3$.

Near each singularity $a_{i}$ the lifted vectorfields $\tau_{1}, \tau_{2}, \tau_{3}$ are also orthonormal. In fact, for any point $x \in \Sigma \cap \mathbb{B}_{\delta_{0}}\left(a_{i}\right), \frac{x_{0}-a_{i 0}}{\left|x-a_{i}\right|}=p_{0}$, and

$$
\tau_{1}(x)=\left(-\sqrt{1-p_{0}^{2}}, \frac{p_{0}}{\sqrt{1-p_{0}^{2}}} \frac{x_{1}-a_{i 1}}{\left|x-a_{i}\right|}, \frac{p_{0}}{\sqrt{1-p_{0}^{2}}} \frac{x_{2}-a_{i 2}}{\left|x-a_{i}\right|}, \frac{p_{0}}{\sqrt{1-p_{0}^{2}}} \frac{x_{3}-a_{i 3}}{\left|x-a_{i}\right|}\right) .
$$

Also $\tau_{1}(x), \tau_{2}(x), \tau_{3}(x)$ are orthonormal for such $x$ because the Hopf map is horizontally orthogonal and the lifts $\tau_{2}(x), \tau_{3}(x)$ are tangent to the 3 sphere $\left\{p_{0}\right\} \times \sqrt{1-p_{0}^{2}} \mathbb{S}^{3}$.

On the remainder of the surface $\Sigma \backslash \cup_{i=1}^{m} \mathbb{B}_{\delta_{0}}\left(a_{i}\right)$, the linearly independent vectorfields $\tau_{1}, \tau_{2}, \tau_{3}$ are not necessarily orthonormal, and we use their Gram-Schmidt orthonormalizations

$$
\begin{aligned}
& \tilde{\tau}_{1}=\frac{\tau_{1}}{\left|\tau_{1}\right|} \\
& \tilde{\tau}_{2}=\frac{\tau_{2}-\left(\tilde{\tau}_{1} \cdot \tau_{2}\right) \tilde{\tau}_{1}}{\left|\tau_{2}-\left(\tilde{\tau}_{1} \cdot \tau_{2}\right) \tilde{\tau}_{1}\right|}=\frac{\tau_{2}-\left(\tilde{\tau}_{1} \cdot \tau_{2}\right) \tilde{\tau}_{1}}{\left|\tilde{\tau}_{1} \wedge \tau_{2}\right|} \\
& \tilde{\tau}_{3}=\frac{\tau_{3}-\left(\tilde{\tau}_{1} \cdot \tau_{3}\right) \tilde{\tau}_{1}-\left(\tilde{\tau}_{2} \cdot \tau_{3}\right) \tilde{\tau}_{2}}{\left|\tau_{3}-\left(\tilde{\tau}_{1} \cdot \tau_{3}\right) \tilde{\tau}_{1}-\left(\tilde{\tau}_{2} \cdot \tau_{3}\right) \tilde{\tau}_{2}\right|}=\frac{\tau_{3}-\left(\tilde{\tau}_{1} \cdot \tau_{3}\right) \tilde{\tau}_{1}-\left(\tilde{\tau}_{2} \cdot \tau_{3}\right) \tilde{\tau}_{2}}{\left|\tilde{\tau}_{1} \wedge \tilde{\tau}_{2} \wedge \tau_{3}\right|}
\end{aligned}
$$

which provide an orthonormal framing for the unit normal bundle of $\Sigma$.

We need to estimate the total variation of these orthonormalizations. Noting that $\left|\nabla\left(\frac{\tau}{|\tau|}\right)\right| \leq 2 \frac{|\nabla \tau|}{|\tau|}$ for any differentiable $\tau$, we see that

$$
\begin{aligned}
& \left|\nabla \tilde{\tau}_{1}\right| \leq 2 \frac{\left|\nabla \tau_{1}\right|}{\left|\tau_{1}\right|} \leq 2 \frac{\left|\nabla \tau_{1}\right|\left|\tau_{1}\right|\left|\tau_{2}\right|\left|\tau_{3}\right|}{\left|\tau_{1}\right|\left|\tau_{1} \wedge \tau_{2} \wedge \tau_{3}\right|}=2 \frac{\left|\tau_{2}\right|\left|\tau_{3}\right|\left|\nabla \tau_{1}\right|}{\left|\tau_{1} \wedge \tau_{2} \wedge \tau_{3}\right|}, \\
& \left|\nabla \tilde{\tau}_{2}\right|=2\left[\frac{\tau_{2}-\left(\tilde{\tau}_{1} \cdot \tau_{2}\right) \tilde{\tau}_{1}}{\left|\tilde{\tau}_{1} \wedge \tau_{2}\right|}\right] \leq 2\left[\frac{2\left|\nabla \tau_{2}\right|+2\left|\tau_{2}\right|\left|\nabla \tilde{\tau}_{1}\right|}{\left|\tau_{1} \wedge \tau_{2}\right|\left|\tau_{1}\right|^{-1}}\right] \\
& \leq 8\left[\frac{\left|\tau_{1}\right|\left|\nabla \tau_{2}\right|+\left|\tau_{2}\right|\left|\nabla \tau_{1}\right|}{\left|\tau_{1} \wedge \tau_{2}\right|} \cdot \frac{\left|\tau_{1} \wedge \tau_{2}\right|\left|\tau_{3}\right|}{\left|\tau_{1} \wedge \tau_{2} \wedge \tau_{3}\right|}\right]=8\left[\frac{\left|\tau_{2}\right|\left|\tau_{3}\right|\left|\nabla \tau_{1}\right|+\left|\tau_{1}\right|\left|\tau_{3}\right|\left|\nabla \tau_{2}\right|}{\left|\tau_{1} \wedge \tau_{2} \wedge \tau_{3}\right|}\right], \\
& \left|\nabla \tilde{\tau}_{3}\right| \leq 2\left[\frac{3\left|\nabla \tau_{3}\right|+2\left|\tau_{3}\right|\left|\nabla \tilde{\tau}_{1}\right|+2\left|\tau_{3}\right|\left|\nabla \tilde{\tau}_{2}\right|}{\left|\tilde{\tau}_{1} \wedge \tilde{\tau}_{2} \wedge \tau_{3}\right|}\right] \\
& \leq 32\left[\frac{\left|\nabla \tau_{3}\right|+\left|\tau_{3}\right|\left|\tau_{1}\right|^{-1}\left|\nabla \tau_{1}\right|+\left|\tau_{3}\right|\left(\frac{\left|\tau_{1}\right|\left|\nabla \tau_{2}\right|+\left|\tau_{2}\right|\left|\nabla \tau_{1}\right|}{\left|\tau_{1} \wedge \tau_{2}\right|}\right)}{\left|\frac{\tau_{1}}{\left|\tau_{1}\right|} \wedge\left(\frac{\tau_{2}}{\left.|| \tau_{1}\right|^{-1} \tau_{1} \wedge \tau_{2} \mid}\right) \wedge \tau_{3}\right|}\right] \\
& \leq 32\left[\frac{\left|\tau_{1}\right|\left|\tau_{2}\right|\left|\nabla \tau_{3}\right|+\left|\tau_{2}\right|\left|\tau_{3}\right|\left|\nabla \tau_{1}\right|+\left|\tau_{1}\right|\left|\tau_{3}\right|\left|\nabla \tau_{2}\right|}{\left|\tau_{1} \wedge \tau_{2} \wedge \tau_{3}\right|}\right] \text {. }
\end{aligned}
$$

Inasmuch as

$$
\left|\tau_{j}\right| \leq|\nabla u|, \quad\left|\nabla \tau_{j}\right| \leq\left|\nabla^{2} u\right|, \quad\left|\tau_{1} \wedge \tau_{2} \wedge \tau_{3}\right|=J_{3} u,
$$


we deduce the general pointwise estimate

$$
\left|\nabla \tilde{\tau}_{j}\right| \leq c \frac{|\nabla u|^{2}\left|\nabla^{2} u\right|}{\left|J_{3} u\right|} \leq c \frac{|\nabla u|^{4}+\left|\nabla^{2} u\right|^{2}}{\left|J_{3} u\right|},
$$

which we may integrate using (IV.27) to obtain the variation estimate along $\Sigma=u^{-1}\{p\}$,

$$
\int_{\Sigma}\left|\nabla \tilde{\tau}_{j}\right| d \mathcal{H}^{2} \leq c \int_{\mathbb{B}^{5}}\left|\nabla^{2} u\right|^{2} d x
$$

\section{IV.3 Twisting of the Normal Frame $\tilde{\tau}_{1}, \tilde{\tau}_{2}, \tilde{\tau}_{3}$ About Each Singularity $a_{i}$}

First we recall from[MS, $\S 5-6$ that the Grassmannian

$$
\tilde{G}_{2}\left(\mathbb{R}^{5}\right)
$$

of oriented 2 planes through the origin in $\mathbb{R}^{5}$ is a compact smooth manifold of dimension 6 . It may be identified with the set of simple unit 2 vectors in $\mathbb{R}^{5}$,

$$
\left\{v \wedge w \in \wedge_{2} \mathbb{R}^{5}: v \in \mathbb{S}^{4}, w \in \mathbb{S}^{4}, v \cdot w=0\right\} .
$$

We will use the distance $|P-Q|$ on $\tilde{G}_{2}\left(\mathbb{R}^{5}\right)$ given by this embedding into $\wedge_{2} \mathbb{R}^{5} \approx \mathbb{R}^{10}$.

For a fixed plane $P \in \tilde{G}_{2}\left(\mathbb{R}^{5}\right)$, the set of nontransverse 2 planes

$$
\mathcal{Q}_{P}=\left\{Q \in \tilde{G}_{2}\left(\mathbb{R}^{5}\right): P \cap Q \neq\{0\}\right\}
$$

is a (Schubert) subvariety of dimension $1+3=4$ because every $Q \in \mathcal{Q}_{P} \backslash\{P\}$ equals $v \wedge w$ for some $w \in \mathbb{S}^{4} \cap P$ and some $v \in \mathbb{S}^{4} \cap w^{\perp}$. These subvarieties are all orthogonally isomorphic and, in particular, have the same finite 4 dimensional Hausdorff measure. Also

$$
Y_{P}=\left\{Q \in \mathcal{Q}_{P}: P^{\perp} \cap Q \neq\{0\}\right\}
$$

is a closed subvariety of dimension 3 , and $\mathcal{Q}_{P} \backslash Y_{P}$ is a smooth submanifold.

Then, near each singularity $a_{i}$, the set of 2 planes nontransverse to the cone $\Sigma \cap \mathbb{B}_{\delta_{0}}\left(a_{i}\right) \backslash\left\{a_{i}\right\}$,

$$
W=\bigcup_{x \in \Sigma \cap \mathbb{B}_{\delta_{0}}\left(a_{i}\right) \backslash\left\{a_{i}\right\}} \mathcal{Q}_{\operatorname{Tan}(\Sigma, x)}=\bigcup_{x \in \Gamma_{i}} \mathcal{Q}_{\operatorname{Tan}(\Sigma, x)},
$$

has dimension only $1+4=5<6=\operatorname{dim} \tilde{G}_{2}\left(\mathbb{R}^{5}\right)$. Note also its location, that $W$ is, by the smallness of $\left|p_{0}\right|$, contained in the tubular neighborhood

$$
V \equiv\left\{Q \in \tilde{G}_{2}\left(\mathbb{R}^{5}\right): \operatorname{dist}\left(Q, \tilde{G}_{2}\left(\{0\} \times \mathbb{R}^{4}\right)<1 / 50\right\},\right.
$$

of the 4 dimensional subgrassmannian $\tilde{G}_{2}\left(\{0\} \times \mathbb{R}^{4}\right)$.

We now describe explicitly how the framing $\tilde{\tau}_{1}(x), \tilde{\tau}_{2}(x), \tilde{\tau}_{3}(x)$ twists once as $x$ goes around each circle $\Gamma_{i}$. The problem is that the vectors $\tilde{\tau}_{j}(x)$ lie in the normal space $\operatorname{Nor}(\Sigma, x)$ which also varies with $x$. To measure the rotation of the frame $\tilde{\tau}_{1}(x), \tilde{\tau}_{2}(x), \tilde{\tau}_{3}(x)$, as $x$ traverses the circle $\Gamma_{i}$, it is necessary to use some reference frame for $\operatorname{Nor}(\Sigma, x)$. 
We can induce such a frame from some fixed unit vectors in $\mathbb{R}^{5}$ as follows: Consider a fixed $Q \in$ $\tilde{G}_{2}\left(\mathbb{R}^{5}\right) \backslash W$, and suppose $Q=v \wedge w$ with $v, w$ being an orthonormal basis for $Q$. For each $x \in \Gamma_{i}$, the orthogonal projections of $v, w$ onto $\operatorname{Nor}(\Sigma, x)$ are linearly independent; let $\sigma_{1}(x), \sigma_{2}(x)$ be their GramSchmidt orthonormalizations. We then get $\sigma_{3}(x)$ by using the map $u$ to pull-back the orientation of $\mathbb{S}^{3}$ to $\operatorname{Nor}(\Sigma, x)$ so that the resulting orienting 3 vector is $\sigma_{1}(x) \wedge \sigma_{2}(x) \wedge \sigma_{3}(x)$ for a unique unit vector $\sigma_{3}(x) \in \operatorname{Nor}(\Sigma, x)$ orthogonal to $\sigma_{1}(x), \sigma_{2}(x)$. We view

$$
\sigma_{1}(x), \sigma_{2}(x), \sigma_{3}(x)
$$

as the reference frame determined by the fixed vectors $v, w$. For each $x \in \Gamma_{i}$, there is then a unique rotation $\gamma(x) \in \mathbb{S O}(3)$ so that

$$
\gamma(x)\left[\sigma_{j}(x)\right]=\tilde{\tau}_{j}(x) \text { for } j=1,2,3 .
$$

In the next paragraph we will check that $\gamma: \Gamma_{i} \rightarrow \mathbb{S O}(3)$ is a single geodesic circle in $\mathbb{S O}(3)$. The twisting of the frame $\tilde{\tau}_{1}, \tilde{\tau}_{2}, \tilde{\tau}_{3}$ around the circle $\Gamma_{i}$ is reflected in the fact that such a circle induces the nonzero element in $\Pi_{1}(\mathbb{S O}(3)) \simeq \mathbb{Z}_{2}$.

In the special case $v=(1,0,0,0)$, the normalized orthogonal projection of $v$ onto $\operatorname{Nor}(\Sigma, x)$ is, by (IV.4), simply

$$
\sigma_{1}(x)=\tilde{\tau}_{1}(x) .
$$

So in this case, each orthogonal matrix $\gamma(x)$ is a rotation about the first axis, and one checks that, as $x$ traverses the circle $\Gamma_{i}$ once, these rotations complete a single geodesic circle in $\mathbb{S O}(3)$. For another choice of $v$, the geodesic circle $\gamma: \Gamma_{i} \rightarrow \mathbb{S O}(3)$ involves a circle of rotations about a different axis combined with a single orthogonal change of coordinates.

\section{IV.4 A Reference Normal Framing for $\Sigma=u^{-1}\{p\}$}

The above calculations near the $a_{i}$ suggest comparing on the whole surface $u^{-1}\{p\}$ the pull-back normal framing $\tilde{\tau}_{1}(x), \tilde{\tau}_{2}(x), \tilde{\tau}_{3}(x)$ with some reference normal framing $\sigma_{1}(x), \sigma_{2}(x), \sigma_{3}(x)$ induced by two fixed vectors $v, w$. Unfortunately, there may not exist fixed vectors $v, w$ so that the corresponding reference framing $\sigma_{1}, \sigma_{2}, \sigma_{3}$ is defined everywhere on $\Sigma$. In this section we show that any orthonormal basis $v, w$ of almost every oriented 2 plane $Q \in \tilde{G}_{2}\left(\mathbb{R}^{5}\right)$ gives a reference framing on $\Sigma$ which is well-defined and smooth except at finitely many discontinuities

$$
b_{1}, b_{2}, \ldots, b_{n} .
$$

We will then need to connect the original singularities $a_{i}$ to the $b_{j}$ (or to $\partial \mathbb{B}^{5}$ ) and, in $9 \mathrm{IV} .6$, choose other curves to connect the $b_{j}$ to each other (or to $\partial \mathbb{B}^{5}$ ), with all curves having total length bounded by a multiple of $\int_{\mathbb{B}^{5}}\left|\nabla^{2} u\right|^{2} d x$.

To find a suitable $Q=v \wedge w$, we will first rule out the exceptional planes that contain some nonzero vector normal to $\Sigma$ at some point $x \in \Sigma$. The really exceptional 2 planes that lie completely in some normal space

$$
X=\cup_{x \in \Sigma} X_{x} \quad \text { where } \quad X_{x}=\left\{Q \in \tilde{G}_{2}\left(\mathbb{R}^{5}\right): Q \subset \operatorname{Nor}(\Sigma, x)\right\} .
$$

Then $X$ has dimension at most $2+2=4<6=\operatorname{dim} \tilde{G}_{2}\left(\mathbb{R}^{5}\right)$ because $\operatorname{dim} \Sigma=2$ and $\operatorname{dim} \tilde{G}_{2}\left(\mathbb{R}^{3}\right)=2$. The remaining set of exceptional planes

$$
Y=\cup_{x \in \Sigma} Y_{x} \quad \text { where } \quad Y_{x}=\left\{Q \in \tilde{G}_{2}\left(\mathbb{R}^{5}\right): \operatorname{dim}(Q \cap \operatorname{Nor}(\Sigma, x))=1\right\}
$$

has dimension at most $2+2+1=5<6=\operatorname{dim} \tilde{G}_{2}\left(\mathbb{R}^{5}\right)$ because

$$
Y_{x}=\left\{e \wedge w: e \in \mathbb{S}^{4} \cap \operatorname{Nor}(\Sigma, x) \text { and } w \in \mathbb{S}^{4} \cap \operatorname{Tan}(\Sigma, x)\right\} .
$$


In terms of our previous notation, $Y_{\operatorname{Tan}(\Sigma, x)}=X_{x} \cup Y_{x}$.

Any unit vector $e \notin \operatorname{Nor}(\Sigma, x)$ has a nonzero orthogonal projection

$$
e_{T}(x)
$$

onto $\operatorname{Tan}(\Sigma, x)$.

Normalizing

$$
\tilde{e}_{T}(x)=\frac{e_{T}(x)}{\left|e_{T}(x)\right|},
$$

we find a unique unit vector $e_{\Sigma}(x) \in \operatorname{Tan}(\Sigma, x)$ orthogonal to $e_{T}(x)$ so that $\tilde{e}_{T}(x) \wedge e_{\Sigma}(x)$ is the standard orientation of $\operatorname{Tan}(\Sigma, x)$. Then

$$
e \cdot e_{\Sigma}(x)=\left(e-e_{T}(x)\right) \cdot e_{\Sigma}(x)+e_{T}(x) \cdot e_{\Sigma}(x)=0+0
$$

because $e-e_{T}(x) \in \operatorname{Nor}(\Sigma, x)$. Thus,

$$
\tilde{e}_{T}(x), e_{\Sigma}(x), \tilde{\tau}_{1}(x), \tilde{\tau}_{2}(x), \tilde{\tau}_{3}(x)
$$

is an orthonormal basis for $\mathbb{R}^{5}$.

Away from the 4 dimensional unit normal bundle

$$
\mathcal{N}_{\Sigma}=\left\{(x, e): x \in \Sigma, e \in \mathbb{S}^{4} \cap \operatorname{Nor}(\Sigma, x)\right\},
$$

we now define the basic map

$$
\Phi:\left(\Sigma \times \mathbb{S}^{4}\right) \backslash \mathcal{N}_{\Sigma} \rightarrow G_{2}\left(\mathbb{R}^{5}\right), \quad \Phi(x, e)=e \wedge e_{\Sigma}(x)
$$

to parameterize the planes nontransverse to $\Sigma$ in $\tilde{G}_{2}\left(\mathbb{R}^{5}\right) \backslash Y$. Incidentally, these do include the 2 dimensional family of tangent planes

$$
Z=\left\{Q \in \tilde{G}_{2}\left(\mathbb{R}^{5}\right): Q=\operatorname{Tan}(\Sigma, x) \text { for some } x \in \Sigma\right\} .
$$

In terms of the notation at the beginning of this section, for any 2 plane $Q \notin Y$,

$$
Q \in \mathcal{Q}_{\operatorname{Tan}(\Sigma, x)} \Longleftrightarrow Q=\Phi(x, e) \text { for some } e \in \mathbb{S}^{4} \backslash \operatorname{Nor}(\Sigma, x) \text {. }
$$

Note that $\Phi(x,-e)=\Phi(x, e)$, and, in fact,

$$
\Phi\left(x, e^{\prime}\right)=\Phi(x, e) \in \tilde{G}_{2}\left(\mathbb{R}^{5}\right) \backslash Y \quad \Longleftrightarrow \quad e^{\prime}= \pm e .
$$

It is also easy to describe the behavior of $\Phi$ at the singular set $\mathcal{N}_{\Sigma}$. A 2 plane $Q$ belongs to $Y$, that is, $Q=v \wedge w$ for some $v \in \operatorname{Nor}(\Sigma, x) \cap \mathbb{S}^{4}$ and $w \in \operatorname{Tan}(\Sigma, x) \cap \mathbb{S}^{4}$, if and only if $Q=\lim _{n \rightarrow \infty} \Phi\left(x_{n}, v_{n}\right)$ for some sequence $\left(x_{n}, v_{n}\right) \in\left(\Sigma \times \mathbb{S}^{4}\right) \backslash \mathcal{N}_{\Sigma}$ approaching $(x, v)$. The map $\Phi$ essentially "blows-up" the 4 dimensional $\mathcal{N}_{\Sigma}$ to the 5 dimensional $Y$, and, in particular, any smooth curve in $\tilde{G}_{2}\left(\mathbb{R}^{5}\right)$ transverse to $Y$ lifts by $\Phi$ to a pair of antipodal curves in $\Sigma \times \mathbb{S}^{4}$ extending continuously transversally across $\mathcal{N}_{\Sigma}$.

We now choose and fix $Q \in G^{2}\left(\mathbb{R}^{5}\right)$ so that neither $Q$ nor $-Q$ belong to the 5 dimensional exceptional set $X \cup Y \cup Z$ and both are regular values of $\Phi$. We may also insist that $Q$ is close to the 3 dimensional Schubert cycle

$$
H=\left\{(1,0, \ldots, 0) \wedge\left(0, v_{1}, v_{2}, v_{3}, v_{4}\right): v_{1}^{2}+\cdots+v_{4}^{2}=1\right\},
$$

say $\operatorname{dist}(Q, H)<1 / 100$. This will guarantee that $Q$ is well separated from the open region $V$ that contains $W$. 
Since

$$
\operatorname{dim}\left(\Sigma \times \mathbb{S}^{4}\right)=6=\operatorname{dim} \tilde{G}_{2}\left(\mathbb{R}^{5}\right)
$$

$\Phi^{-1}\{Q,-Q\}$ is a finite set, say

$$
\Phi^{-1}\{Q,-Q\}=\left\{\left(b_{1}, e_{1}\right),\left(b_{1},-e_{1}\right),\left(b_{2}, e_{2}\right),\left(b_{2},-e_{2}\right), \ldots,\left(b_{n}, e_{n}\right),\left(b_{n},-e_{n}\right)\right\} .
$$

We now see that the reference framing $\sigma_{1}(x), \sigma_{2}(x), \sigma_{3}(x)$ of $\operatorname{Nor}(\Sigma, x)$ corresponding to any fixed orthonormal basis $v, w$ of $Q$ fails to exist precisely at the points $b_{1}, b_{2}, \ldots, b_{n}$. As before, we now have the smooth mapping

$$
\gamma: \Sigma \backslash\left\{a_{1}, \ldots, a_{m}, b_{1}, \ldots, b_{n}\right\} \rightarrow \mathbb{S O}(3),
$$

which is defined by the condition $\gamma(x)\left[\sigma_{j}(x)\right]=\tilde{\tau}_{j}(x)$ for $j=1,2,3$ or, in column-vector notation,

$$
\gamma=\left[\sigma_{1} \sigma_{2} \sigma_{3}\right]^{-1}\left[\tilde{\tau}_{1} \tilde{\tau}_{2} \tilde{\tau}_{3}\right]
$$

\section{IV.5 Asymptotic Behavior of $\gamma$ Near the Singularities $a_{i}$ and $b_{j}$}

As discussed in $\S 3.1$, the map $u$, the surface $\Sigma=u^{-1}\{p\}$, the frames $\tilde{\tau}_{1}, \tilde{\tau}_{2}, \tilde{\tau}_{3}$ and $\sigma_{1}, \sigma_{2}, \sigma_{3}$, and the rotation field $\gamma$ are all precisely known near a singularity $a_{i}$ in the cone neighborhood $\Sigma \cap \mathbb{B}_{\delta_{0}}\left(a_{i}\right) \backslash\left\{a_{i}\right\}$. In particular, $\gamma$ is homogeneous of degree 0 on $\Sigma \cap \mathbb{B}_{\delta_{0}}\left(a_{i}\right) \backslash\left\{a_{i}\right\}$; on its boundary $\gamma \mid \Gamma_{i}$ is a constant-speed geodesic circle.

At each $b_{j}$, the frame $\tilde{\tau}_{1}, \tilde{\tau}_{2}, \tilde{\tau}_{3}$ is smooth, but the frame $\sigma_{1}, \sigma_{2}, \sigma_{3}$, and hence the rotation $\gamma$, has an essential discontinuity. Nevertheless, we may deduce some of the asymptotic behavior at $b_{j}$ because $\pm Q$ were chosen to be regular values of $\Phi$. In fact, we'll verify:

The tangent map $\gamma_{j}$ of $\gamma$ at $b_{j}$,

$$
\gamma_{j}: \operatorname{Tan}\left(\Sigma, b_{j}\right) \cap \mathbb{B}_{1}(0) \rightarrow \mathbb{S O}(3), \quad \gamma_{j}(x)=\lim _{r \rightarrow 0} \gamma\left[\exp _{b_{j}}^{\Sigma}(r x)\right],
$$

exists and is the homogeneous degree 0 extension of some reparameterization of a geodesic circle in $\mathbb{S O}(3)$. In particular, for small positive $\delta, \gamma \mid\left(\Sigma \cap \partial \mathbb{B}_{\delta}\left(b_{j}\right)\right)$ is an embedded circle inducing the nonzero element of $\Pi_{1}(\mathbb{S O}(3)) \simeq \mathbb{Z}_{2}$.

To check this, we use, as above, the more convenient orthonormal basis $\left\{e_{j}, e_{j \Sigma}\right\}$ for $Q$; that is,

$$
e_{j \Sigma}=e_{j \Sigma}\left(b_{j}\right) \in \operatorname{Tan}\left(\Sigma, b_{j}\right) \text { and } Q=e_{j} \wedge e_{j \Sigma}=\Phi\left(b_{j}, \pm e_{j}\right)
$$

Then, for $x \in \Sigma$, let

$$
e_{j}^{N}(x), \quad e_{j \Sigma}^{N}(x)
$$

denote the orthogonal projections of the fixed vectors $e_{j}, e_{j \Sigma}$ onto $\operatorname{Nor}(\Sigma, x)$, and

$$
\hat{e}_{j}^{N}(x)
$$

denote the cross-product of $e_{j \Sigma}^{N}(x)$ and $e_{j}^{N}(x)$ in $\operatorname{Nor}(\Sigma, x)$. These three vectorfields are smooth near $b_{j}$ with

$$
e_{j}^{N}\left(b_{j}\right) \neq 0, \quad e_{j \Sigma}^{N}\left(b_{j}\right)=0, \quad \hat{e}_{j}^{N}\left(b_{j}\right)=0 .
$$

Here our insistence that $\pm Q \notin Z$ guarantees that $Q$ is not tangent to $\Sigma$ at $b_{j}$. Let $g_{j}$ denote the orthogonal projection of $\mathbb{R}^{5}$ onto the 2 plane

$$
P_{j}=\operatorname{Nor}\left(\Sigma, b_{j}\right) \cap\left[e_{j}^{N}\left(b_{j}\right)\right]^{\perp}
$$


Then $G_{j}(x)=g_{j} \circ e_{j}^{N}(x)$ defines a smooth map from a $\Sigma$ neighborhood of $b_{j}$ to $P_{j}$, which has, by the regularity of $\Phi$ at $\left(b_{j}, \tilde{e}_{j}\right.$ ), a simple, nondegenerate zero at $b_{j}$ (of degree \pm 1 ). It follows that as $x$ circulates $\Sigma \cap \partial \mathbb{B}_{\delta}\left(b_{j}\right)$ once, for $\delta$ small, $G_{j}(x)$ and similarly $g_{j} \circ \hat{e}_{j}^{N}(x)$, circulate 0 once in $P_{j}$. Returning to the original basis $v, w$ of $Q$, we now check that, as $x$ circulates $\Sigma \cap \partial \mathbb{B}_{\delta}\left(b_{j}\right)$ once, the frame $\sigma_{1}(x), \sigma_{2}(x), \sigma_{3}(x)$ approximately, and asymptotically as $\delta \rightarrow 0$, rotates once about the vector $e_{j}^{N}\left(b_{j}\right)$. Since the frame $\tilde{\tau}_{1}(x), \tilde{\tau}_{2}(x), \tilde{\tau}_{3}(x)$ is smooth at $b_{j}$, we see that the map $\gamma$ has, at $b_{j}$, a tangent map $\gamma_{j}$ as described above.

\section{IV.6 Connecting the Singularities $a_{i}$ to the $b_{j}$ or to $\partial \mathbb{B}^{5}$}

Here we will find curves reaching all the $a_{i}$ and $b_{j}$. Concerning the $a_{i}$, we recall from $[\mathrm{Br}, \oint I I I, 10]$ that $\mathbb{S O}(3)$ is isometric to $\mathbb{R P}^{3} \simeq \mathbb{S}^{3} /\{x \sim-x\}$. Any geodesic circle $\Gamma$ in $\mathbb{S O}(3)$ generates $\Pi_{1}(\mathbb{S O}(3)) \simeq \mathbb{Z}_{2}$ and lifts to a great circle $\tilde{\Gamma}$ in $\mathbb{S}^{3}$. The rotations at maximal distance from $\Gamma$ form another geodesic circle $\Gamma^{\perp}$ and the nearest point retraction

$$
\rho_{\Gamma}: \mathbb{S O}(3) \backslash \Gamma \rightarrow \Gamma^{\perp}
$$

is induced by the standard nearest point retraction

$$
\rho_{\tilde{\Gamma}}: \mathbb{S}^{3} \backslash \tilde{\Gamma} \rightarrow \tilde{\Gamma}^{\perp}
$$

In particular,

$$
\left|\nabla \rho_{\Gamma}(\zeta)\right| \leq \frac{c}{\operatorname{dist}(\zeta, \Gamma)} \text { for } \zeta \in \mathbb{S O}(3) .
$$

Any geodesic circle $\Gamma^{\prime}$ in $\mathbb{S O}(3)$ that does not intersect $\Gamma$ is mapped diffeomorphically by $\rho_{\Gamma}$ onto the circle $\Gamma^{\perp}$. We deduce that if $\Gamma$ is chosen to miss the asymptotic circles

$$
\gamma\left(\Gamma_{i}\right) \quad \text { and } \quad \gamma_{j}\left(\operatorname{Tan}\left(\Sigma, b_{j}\right) \cap \mathbb{S}^{4}\right)
$$

associated with the singularities $a_{i}$ and $b_{j}$, then, on $\Sigma$, the composition $\rho_{\Gamma} \circ \gamma$ maps every sufficiently small circle

$$
\Sigma \cap \partial \mathbb{B}_{\delta}\left(a_{i}\right) \text { and } \Sigma \cap \partial \mathbb{B}_{\delta}\left(b_{j}\right)
$$

diffeomorphically onto the circle $\Gamma^{\perp}$.

Under the identification of $\mathbb{S O}(3)$ with $\mathbb{R P}^{3}, \mathbb{S O}(4)$ acts transitively by isometry on

$$
\mathcal{G}=\{\text { geodesic circles } \Gamma \subset \mathbb{S O}(3)\} .
$$

Then $\mathcal{G}$ is compact and admits a positive invariant measure $\mu_{\mathcal{G}}$. For $\mu_{\mathcal{G}}$ almost every circle $\Gamma$,

$$
\Gamma \cap \gamma\left(\Gamma_{i}\right)=\emptyset \text { for } i=1, \ldots, m, \quad \Gamma \cap \gamma_{j}\left(\operatorname{Tan}\left(\Sigma, b_{j}\right) \cap \mathbb{S}^{4}\right)=\emptyset \text { for } j=1, \ldots, n,
$$

and $\Gamma$ is transverse to the map $\gamma$. In particular, $\gamma^{-1}(\Gamma)$ is a finite subset

$$
\left\{c_{1}, c_{2}, \ldots, c_{\ell}\right\}
$$

of $\Sigma$. For such a circle $\Gamma$ and any regular value $z \in \Gamma^{\perp}$ of

$$
\rho_{\Gamma} \circ \gamma: \Sigma \backslash\left\{a_{1}, \ldots, a_{m}, b_{1}, \ldots, b_{n}, c_{1}, \ldots, c_{\ell}\right\} \rightarrow \Gamma^{\perp},
$$

the fiber

$$
A=\left(\rho_{\Gamma} \circ \gamma\right)^{-1}\{z\}
$$


is a smooth embedded 1 dimensional submanifold with

$$
(\operatorname{Clos} A) \backslash A \subset\left\{a_{1}, \ldots, a_{m}, b_{1}, \ldots, b_{n}, c_{1}, \ldots, c_{\ell}\right\} \cup \partial \mathbb{B}^{5} .
$$

We also can deduce the local behavior of $A$ near each of the points $a_{i}, b_{j}, c_{k}$. From the above description of the asymptotic behavior of $\gamma$ near $a_{i}$ and $b_{j}$, we see that

$$
\mathbb{B}_{\delta_{0}}\left(a_{i}\right) \cap \operatorname{Clos} A
$$

is simply a single line segment with one endpoint $a_{i}$ while

$$
\mathbb{B}_{\delta}\left(b_{j}\right) \cap \operatorname{Clos} A
$$

is, for $\delta$ sufficiently small, a single smooth segment with one endpoint $b_{j}$. On the other hand,

$$
\mathbb{B}_{\delta}\left(c_{k}\right) \cap \operatorname{Clos} A
$$

is, for $\delta$ sufficiently small, a single smooth segment with an interior point $c_{k}$. To see this, observe that, for the lifted map $\rho_{\tilde{\Gamma}}: \mathbb{S}^{3} \backslash \tilde{\Gamma} \rightarrow \tilde{\Gamma}^{\perp}$ and any point $\tilde{z} \in \tilde{\Gamma}^{\perp}$, the fiber $\rho_{\tilde{S}}^{-1}\{z\}$ is an open great hemisphere, centered at $z$, with boundary $\tilde{\Gamma}$. It follows for the downstairs map $\rho_{\Gamma}$ that $\left.E_{z}=\operatorname{Clos}\left(\rho_{\Gamma}^{-1}\{z\}\right)\right\}$ is a full geodesic 2 sphere containing $z$ and the circle $\Gamma$. Since the surface $\gamma(\Sigma)$ intersects the circle $\Gamma$ transversely at a finite set, this sphere $E_{z}$ is also transverse to $\gamma(\Sigma)$ near this set. Thus, for $\delta$ sufficiently small, $\mathbb{B}_{\delta}\left(c_{k}\right) \cap$ Clos $A$, being mapped diffeomorphically by $\gamma$ onto the intersection $E_{z} \cap \gamma\left(\Sigma \cap \mathbb{B}_{\delta}\left(c_{k}\right)\right)$, is an open smooth segment containing $c_{k}$ in its interior.

Combining this boundary behavior with the interior smoothness of the 1 manifold $A$, we now conclude that

$\mathbb{B}^{5} \cap$ Clos A globally consists of disjoint smooth segments joining pairs of points from

$$
\left\{a_{1}, \ldots, a_{m}, b_{1}, \ldots, b_{n}\right\} \cup \partial \mathbb{B}^{5} .
$$

Moreover, each point $a_{i}$ or $b_{j}$ is the endpoint of precisely one segment.

\section{IV.7 Estimating the Length of the Connecting Set $A$}

The definition of the $A$ depends on many choices:

(1) the point $p \in \mathbb{S}^{3}$ near $\{0\} \times \mathbb{R}^{4}$, which determines the surface $\Sigma=u^{-1}\{p\}$,

(2) the vectors $\eta_{2}, \eta_{2}, \eta_{3} \in \operatorname{Tan}\left(\mathbb{S}^{3}, p\right)$, which determine the pull-back normal framing $\tilde{\tau}_{1}, \tilde{\tau}_{2}, \tilde{\tau}_{3}$,

(3) the vectors $v, w \in \mathbb{S}^{4}$, which determine the reference normal framing $\sigma_{1}, \sigma_{2}, \sigma_{3}$ and the rotation field $\gamma=\left[\sigma_{1} \sigma_{2} \sigma_{3}\right]^{-1}\left[\tilde{\tau}_{1} \tilde{\tau}_{2} \tilde{\tau}_{3}\right]: \Sigma \backslash\left\{b_{1}, \ldots, b_{m}\right\} \rightarrow \mathbb{S O}(3)$

(4) the circle $\Gamma \subset \mathbb{S O}(3)$, which determines the retraction $\rho_{\Gamma}: \mathbb{S O}(3) \backslash \Gamma \rightarrow \Gamma^{\perp}$, and

(5) the point $z \in \Gamma^{\perp}$, which finally gives $A=\left(\rho_{\Gamma} \circ \gamma\right)^{-1}\{z\}$.

We need to make suitable choices of these to get the desired length estimate for $A$. In $₫$ IV.1 we already used one coarea formula to choose $p \in \mathbb{S}^{3}$ to give the basic estimate (IV.25)

$$
\int_{\Sigma} \frac{|\nabla u|^{4}+\left|\nabla^{2} u\right|^{2}}{J_{3} u} d \mathcal{H}^{2} \leq c \int_{\mathbb{B}^{5}}\left|\nabla^{2} u\right|^{2} d x
$$


and the pull-back frame estimate (IV.29)

$$
\int_{\Sigma}\left|\nabla \tilde{\tau}_{j}\right| d \mathcal{H}^{2} \leq c \int_{\mathbb{B}^{5}}\left|\nabla^{2} u\right|^{2} d x
$$

independent of the choice of $\eta_{1}, \eta_{2}, \eta_{3}$, then followed. For the choice of $z \in S^{\perp}$, we want to use another coarea formula, $([\mathrm{Fe}, \S 3.2 .22)$

$$
\int_{\Gamma^{\perp}} \mathcal{H}^{1}\left(\rho_{\Gamma} \circ \gamma\right)^{-1}\{z\} d z=\int_{\Sigma}\left|\nabla\left(\rho_{\Gamma} \circ \gamma\right)\right| d \mathcal{H}^{2}
$$

To bound the righthand integral, we first use the chain rule and (IV.30) for the pointwise estimate

$$
\left|\nabla\left(\rho_{\Gamma} \circ \gamma\right)(x)\right|=\left|\nabla\left(\rho_{\Gamma}\right)(\gamma(x))\right||\nabla \gamma(x)| \leq \frac{c}{\operatorname{dist}(\gamma(x), \Gamma)}|\nabla \gamma(x)| .
$$

Next we observe the finiteness of the integral

$$
C=\int_{\mathcal{G}} \frac{1}{\operatorname{dist}(\zeta, \Gamma)} d \mu_{\mathcal{G}} \Gamma<\infty,
$$

independent of the point $\zeta \in \mathbb{S O}(3)$. To verify this, we note that $\mu_{\mathcal{G}}(\mathcal{G})<\infty$ and choose a smooth coordinate chart for $\mathbb{S O}(3)$ near $\zeta$ that maps $\zeta$ to $0 \in \mathbb{R}^{3}$ and that transforms circles into affine lines in $\mathbb{R}^{3}$. Distances are comparable, and an affine line in $\mathbb{R}^{3} \backslash\{0\}$ is described by its nearest point $a$ to the origin and a direction in the plane $a^{\perp}$. Since

$$
\mu_{\mathcal{G}}\{\Gamma \in \mathcal{G}: \zeta \in \Gamma\}=0,
$$

the finiteness of $C$ now follows from the finiteness of the 3 dimensional integral

$$
\int_{\mathbb{R}^{3} \cap \mathbb{B}_{1}}|y|^{-1} d y .
$$

We deduce from Fubini's Theorem, (IV.31), and (IV.32) that

$$
\begin{aligned}
\int_{\mathcal{G}} \int_{\Gamma^{\perp}} \mathcal{H}^{1}\left(\rho_{\Gamma} \circ \gamma\right)^{-1}\{z\} d z d \mu_{\mathcal{G}} \Gamma & \leq c \int_{\Sigma}|\nabla \gamma(x)| \int_{\mathcal{G}} \frac{1}{\operatorname{dist}(\gamma(x), \Gamma)} d \mu_{\mathcal{G}} \Gamma d \mathcal{H}^{2} x \\
& \leq c C \int_{\Sigma}|\nabla \gamma(x)| d \mathcal{H}^{2} x
\end{aligned}
$$

Thus there exists a $\Gamma \in \mathcal{G}$ and $z \in \Gamma^{\perp}$ so that

$$
\mathcal{H}^{1}\left(\rho_{\Gamma} \circ \gamma\right)^{-1}\{z\} \leq c \int_{\Sigma}|\nabla \gamma(x)| d \mathcal{H}^{2} x
$$

To estimate the righthand side, recall the matrix formula

$$
\gamma=\left[\sigma_{1} \sigma_{2} \sigma_{3}\right]^{-1}\left[\tilde{\tau}_{1} \tilde{\tau}_{2} \tilde{\tau}_{3}\right] .
$$

and use Cramer's rule and the product and quotient rules to deduce the pointwise bound

$$
|\nabla \gamma(x)| \leq c \sum_{j=1}^{3}\left(\left|\nabla \sigma_{j}(x)\right|+\left|\nabla \tilde{\tau}_{j}(x)\right|\right) .
$$


In light of (IV.29), it remains to bound each term $\int_{\Sigma}\left|\nabla \sigma_{j}(x)\right| d \mathcal{H}^{2} x$ for $j=1,2,3$.

For the first one, note that

$$
\left|\nabla \sigma_{1}\right|=\left|\nabla\left(\frac{v^{N}}{\left|v^{N}\right|}\right)\right| \leq 2 \frac{\left|\nabla v^{N}\right|}{\left|v^{N}\right|}
$$

where $v^{N}(x)$ is the orthogonal projection of $v$ onto the normal space $\operatorname{Nor}(\Sigma, x)$ for each $x \in \Sigma$. The formula

$$
v^{N}=\sum_{j=1}^{3}\left(v \cdot \tilde{\tau}_{j}\right) \tilde{\tau}_{j}
$$

and the product rule give the pointwise estimate for the numerator,

$$
\left|\nabla v^{N}\right| \leq c \sum_{j=1}^{3}\left|\nabla \tilde{\tau}_{j}\right|
$$

independent of the choice of $v \in \mathbb{S}^{4}$.

To estimate the denominator, we let $v^{L}$ denote the orthogonal projection of $v$ to any fixed 3 dimensional subspace $L$ of $\mathbb{R}^{5}$, and observe the finiteness

$$
C_{1}=\int_{\mathbb{S}^{4}} \frac{1}{\left|v^{L}\right|} d \mathcal{H}^{4} v<\infty,
$$

independent of $L$. To verify this, we note that the projection of $\mathbb{S}^{4}$ to $L$ vanishes along a great circle, and, near any point of this circle, the projection is bilipschitz equivalent to an orthogonal projection of $\mathbb{R}^{4}$ to $\mathbb{R}^{3}$. So the finiteness of $C_{1}$ again follows from the finiteness of the 3 dimensional integral $\int_{\mathbb{R}^{3} \cap \mathbb{B}_{1}}|y|^{-1} d y$.

By Fubini's Theorem, (IV.35), (IV.36), and (IV.29),

$$
\begin{aligned}
\int_{\mathbb{S}^{4}} \int_{\Sigma}\left|\nabla \sigma_{1}(x)\right| d \mathcal{H}^{2} x d \mathcal{H}^{4} v & \leq 2 \int_{\Sigma}\left|\nabla v^{N}(x)\right| \int_{\mathbb{S}^{4}} \frac{1}{\left|v^{N}(x)\right|} d \mathcal{H}^{4} v d \mathcal{H}^{2} x \\
& \leq 2 C_{1} \int_{\Sigma}\left|\nabla v^{N}(x)\right| d \mathcal{H}^{2} x \\
& \leq c \sum_{j=1}^{3} \int_{\Sigma}\left|\nabla \tilde{\tau}_{j}(x)\right| d \mathcal{H}^{2} x \\
& \leq c \int_{\mathbb{B}^{5}}\left|\nabla^{2} u\right|^{2} d x
\end{aligned}
$$

So there exists a $v \in \mathbb{S}^{4}$ giving the $\sigma_{1}$ estimate

$$
\int_{\Sigma}\left|\nabla \sigma_{1}(x)\right| d \mathcal{H}^{2} x \leq c \int_{\mathbb{B}^{5}}\left|\nabla^{2} u\right|^{2} d x
$$

Next we observe that $\sigma_{2}=\frac{w_{2}}{\left|w_{2}\right|}$ where $w_{2}(x)$ is the orthogonal projection onto the 2 dimensional subspace $\operatorname{Nor}(\Sigma, x) \cap \sigma_{1}^{\perp}$. We again find

$$
\left|\nabla \sigma_{2}\right|=\left|\nabla\left(\frac{w_{2}}{\left|w_{2}\right|}\right)\right| \leq 2 \frac{\left|\nabla w_{2}\right|}{\left|w_{2}\right|} .
$$

Now the formula

$$
w_{2}=\left[\sum_{j=1}^{3}\left(w \cdot \tilde{\tau}_{j}\right) \tilde{\tau}_{j}\right]-\left(w \cdot \sigma_{1}\right) \sigma_{1},
$$


and the product rule give the pointwise estimate for the numerator,

$$
\left|\nabla w_{2}\right| \leq c\left(\left|\nabla \sigma_{1}\right|+\sum_{j=1}^{3}\left|\nabla \tilde{\tau}_{j}\right|\right),
$$

independent of the choice $w \in \mathbb{S}^{4}$.

To estimate the denominator, we let $w^{M}$ denote the orthogonal projection of $w$ to any fixed 2 dimensional subspace $M$ of the hyperplane $v^{\perp}=\sigma_{1}^{\perp}$, and observe the finiteness of the integral

$$
C_{2}=\int_{\mathbb{S}^{4} \cap v^{\perp}} \frac{1}{\left|w^{M}\right|} d \mathcal{H}^{3} w<\infty,
$$

independent of the choices of $v$ or $M$. To verify this, we note that the projection of the 3 sphere $\mathbb{S}^{4} \cap v^{\perp}$ to $M$ vanishes along a great circle, where it is now bilipschitz equivalent to an orthogonal projection of $\mathbb{R}^{3}$ to $\mathbb{R}^{2}$. So the finiteness of $C_{2}$ this time follows from the finiteness of the 2 dimensional integral $\int_{\mathbb{R}^{2} \cap \mathbb{B}_{1}}|y|^{-1} d y$.

By Fubini's Theorem, (IV.29), (IV.36), (IV.37), (IV.38) and (IV.39),

$$
\begin{aligned}
\int_{\mathbb{S}^{4} \cap v^{\perp}} \int_{\Sigma}\left|\nabla \sigma_{2}(x)\right| d \mathcal{H}^{2} x d \mathcal{H}^{3} w & \leq 2 \int_{\Sigma}\left|\nabla w_{2}(x)\right| \int_{\mathbb{S}^{4} \cap v^{\perp}} \frac{1}{\left|w_{2}(x)\right|} d \mathcal{H}^{3} w d \mathcal{H}^{2} x \\
& \leq 2 C_{2} \int_{\Sigma}\left|\nabla w_{2}(x)\right| d \mathcal{H}^{2} x \\
& \leq c \int_{\Sigma}\left(\left|\nabla \sigma_{1}(x)\right|+\sum_{j=1}^{3}\left|\nabla \tilde{\tau}_{j}(x)\right|\right) d \mathcal{H}^{2} x \\
& \leq c \int_{\mathbb{B}^{5}}\left|\nabla^{2} u\right|^{2} d x .
\end{aligned}
$$

So there exists a $w \in \mathbb{S}^{4} \cap v^{\perp}$ giving the $\sigma_{2}$ estimate

$$
\int_{\Sigma}\left|\nabla \sigma_{2}(x)\right| d \mathcal{H}^{2} x \leq c \int_{\mathbb{B}^{5}}\left|\nabla^{2} u\right|^{2} d x .
$$

Finally we may use the product rule and the formula

$$
\begin{aligned}
\sigma_{3} & =\left[\left(\sigma_{1} \cdot \tilde{\tau}_{2}\right)\left(\sigma_{2} \cdot \tilde{\tau}_{3}\right)-\left(\sigma_{1} \cdot \tilde{\tau}_{3}\right)\left(\sigma_{2} \cdot \tilde{\tau}_{2}\right)\right] \tilde{\tau}_{1} \\
& +\left[\left(\sigma_{1} \cdot \tilde{\tau}_{3}\right)\left(\sigma_{2} \cdot \tilde{\tau}_{1}\right)-\left(\sigma_{1} \cdot \tilde{\tau}_{1}\right)\left(\sigma_{2} \cdot \tilde{\tau}_{3}\right)\right] \tilde{\tau}_{2} \\
& +\left[\left(\sigma_{1} \cdot \tilde{\tau}_{1}\right)\left(\sigma_{2} \cdot \tilde{\tau}_{2}\right)-\left(\sigma_{1} \cdot \tilde{\tau}_{2}\right)\left(\sigma_{2} \cdot \tilde{\tau}_{1}\right)\right] \tilde{\tau}_{3}
\end{aligned}
$$

along with (IV.29), (IV.37), and (IV.40) to obtain the $\sigma_{3}$ estimate

$$
\int_{\Sigma}\left|\nabla \sigma_{3}(x)\right| d \mathcal{H}^{2} x \leq c \int_{\mathbb{B}^{5}}\left|\nabla^{2} u\right|^{2} d x
$$

Now we may combine (IV.33), (IV.34), (IV.29), (IV.37), (IV.40), and (IV.41) to obtain the desired length estimate

$$
\mathcal{H}^{1}(A)=\mathcal{H}^{1}\left(\rho_{\Gamma} \circ \gamma\right)^{-1}\{z\} \leq c \int_{\mathbb{B}^{5}}\left|\nabla^{2} u\right|^{2} d x
$$




\section{IV.8 Connecting the Singularities $b_{j}$ to $b_{j^{\prime}}$}

Although we now have a good description and length estimate for $A$, we are not done. The problem is that the set Clos $A$ does not necessarily connect each of the original singularities $a_{i}$ to another $a_{i^{\prime}}$ or to $\partial \mathbb{B}^{5}$. The path in Clos $A$ starting at $a_{i}$ may end at some $b_{j}$. To complete the connections between pairs of $a_{i}$, it will be sufficient to find a different union $B$ of curves which connect each frame singularity $b_{j}$ to $\partial \mathbb{B}^{5}$ or to another unique frame singularity $b_{j^{\prime}}$. Then adding to Clos $A$ some components of $B$ will give the desired curves connecting every $a_{i}$ to a distinct $a_{i^{\prime}}$ or to $\partial \mathbb{B}^{5}$. In this section we will use the map $\Phi$ from $₫$ IV.4 to construct this additional connecting set $B$, and we will, in IV.9. obtain the required estimate on the length of $B$.

First we recall the description in $[\mathrm{MS}]$ of $\tilde{G}_{2}\left(\mathbb{R}^{5}\right)$ as a 2 sheeted cover of the Grassmannian of unoriented 2 planes in $R^{5}$. With $Q \in \tilde{G}_{2}\left(\mathbb{R}^{5}\right)$ chosen as before in $₫$ IV.3 consider the 5 dimensional Schubert cycle

$$
\mathcal{S}_{Q}=\left\{P \in \tilde{G}_{2}\left(\mathbb{R}^{5}\right): \operatorname{dim}\left(P \cap Q^{\perp}\right) \geq 1\right\}
$$

and the 4 dimensional subcycle

$$
\mathcal{T}_{Q}=\left\{P \in \tilde{G}_{2}\left(\mathbb{R}^{5}\right): \operatorname{dim}\left(P \cap Q^{\perp}\right) \geq 2\right\}=\left\{P \in \tilde{G}_{2}\left(\mathbb{R}^{5}\right): P \subset Q^{\perp}\right\}
$$

As in $\mathrm{MS}$, we see that $\mathcal{S}_{Q} \backslash \mathcal{T}_{Q}$ is a smooth embedded open 5 dimensional submanifold of $\tilde{G}_{2}\left(\mathbb{R}^{5}\right)$ and that $\tilde{G}_{2}\left(\mathbb{R}^{5}\right) \backslash \mathcal{S}_{Q}$ consists of two open 6 dimensional antipodal cells, $D_{+}$centered at $Q$ and $D_{-}$centered at $-Q$.

Next we will carefully define a (nearest-point) retraction map

$$
\Pi_{\mathbb{Q}}: \tilde{G}_{2}\left(\mathbb{R}^{5}\right) \backslash\{Q,-Q\} \rightarrow \mathcal{S}_{Q}
$$

For $P \in D_{+} \backslash\{Q\}$, there is a unique vector $v \in P \cap \mathbb{S}^{4}$ which is at maximal distance in $P \cap \mathbb{S}^{4}$ from $Q \cap \mathbb{S}^{4}$ and a unique vector $w$ in $Q \cap \mathbb{S}^{4}$ that is closest to $v$; in particular, $0<w \cdot v<1$. Choose $A_{P} \in \operatorname{so}(5)$ so that the corresponding rotation $\exp A_{P} \in S O(5)$ maps $w$ to $v$ and maps $\tilde{w}$ to $\tilde{v}$ where $P=v \wedge \tilde{v}$ and $Q=w \wedge \tilde{w}$. Thus $\exp A_{P}$ maps $Q$ to $P$, preserving orientation. Here $\left(\exp t A_{P}\right)(w)$ defines a geodesic circle in $\mathbb{S}^{4}$, and

$$
t_{P} \equiv \inf \left\{t>0: w \cdot\left(\exp t A_{P}\right)(w)=0\right\}>1
$$

Then $\left(\exp 2 t_{p} A_{P}\right)(w)=-w$ and $\exp 4 t_{p} A_{P}=$ id. It follows that, in $\tilde{G}_{2}\left(\mathbb{R}^{5}\right)$, as $t$ increases,

$$
\begin{gathered}
\left(\exp t A_{P}\right)(Q) \in D_{+} \text {for } 0 \leq t_{P} \quad \text { and } \quad\left(\exp t A_{P}\right)(Q) \in D_{-} \text {for } t_{P}<t \leq 2 t_{P}, \\
\left(\exp 0 A_{P}\right)(Q)=Q, \quad\left(\exp A_{P}\right)(Q)=P, \quad\left(\exp t_{p} A_{P}\right)(Q) \in \mathcal{S}_{Q}, \quad\left(\exp 2 t_{p} A_{P}\right)(Q)=-Q,
\end{gathered}
$$

and we let $\Pi_{Q}(P)=\left(\exp t_{p} A_{P}\right)(Q)$.

As $P$ approaches $\partial D_{+}=\mathcal{S}_{Q}, t_{P} \downarrow 1$ and $\left|\Pi_{Q}(P)-P\right| \rightarrow 0$. Thus, let

$$
\Pi_{Q}(P)=P \text { for } P \in \mathcal{S}_{Q} .
$$

Also, let

$$
\Pi_{Q}(P)=-\Pi_{Q}(-P) \text { for } P \in D_{-} \backslash\{-Q\} .
$$

Next recall that the small tubular neighborhood $V$ of the 4 dimensional subgrassmannian $\tilde{G}_{2}\left(\{0\} \times \mathbb{R}^{4}\right)$ was well-separated from $Q$. It follows that $\Pi_{Q}(V)$ is in a small neighborhood of the 4 dimensional cycle $\Pi_{Q}\left(\tilde{G}_{2}\left(\{0\} \times \mathbb{R}^{4}\right)\right)$. In particular, the 5 dimensional measure of $\Pi_{Q}(V)$ is small, and one easily finds $P \in \mathcal{S}_{Q} \backslash \Pi_{Q}(V)$ so that $p \notin \Pi_{Q}(W)$. 
For $P \in \mathcal{S}_{Q} \backslash \mathcal{T}_{Q}$, the intersection $P \cap Q^{\perp} \cap \mathbb{S}^{4}$ consists of 2 antipodal points in $P \cap \mathbb{S}^{4}$ that are uniquely of maximal distance from $Q \cap \mathbb{S}^{4}$, and one sees that

$$
\mathrm{Clos} \Pi_{Q}^{-1}\{P\}
$$

contains a single semi-circular geodesic arc joining $Q$ and $-Q$. For almost all $P \in \mathcal{S}_{Q} \backslash \mathcal{T}_{Q}$, this semi-circle meets transversely both $Y$, and, near $\pm Q$, each small surface

$$
\Phi\left(\left[\Sigma \cap \mathbb{B}_{\delta}\left(b_{j}\right)\right] \times\left\{e_{j}\right\}\right) .
$$

We will choose $P \in \mathcal{S}_{Q} \backslash \mathcal{T}_{Q}$ also to be a regular value of $\Pi_{Q} \circ \Phi$. Since, near $P, \mathcal{S}_{Q}$ is is a smooth transverse (in fact, orthogonal) to $\Pi_{Q}^{-1}\{P\}$, we find, using IV.4 that the set

$$
\left(\Pi_{Q} \circ \Phi\right)^{-1}\{P\}=\Phi^{-1}\left(\Pi_{Q}^{-1}\{P\}\right)
$$

is an embedded 1 dimensional submanifold, containing $\left\{\left(b_{1}, \pm e_{1}\right), \ldots,\left(b_{m}, \pm e_{m}\right)\right\}$. In small neighborhoods of any two points $\left(b_{j}, e_{j}\right),\left(b_{j},-e_{j}\right)$ the set $\operatorname{Clos}\left(\Pi_{Q} \circ \Phi\right)^{-1}\{P\}$ consists of two smooth segments (antipodal in the $\mathbb{S}^{4}$ factor) which both project, under the projection

$$
p_{\Sigma}: \Sigma \times \mathbb{S}^{4} \rightarrow \Sigma,
$$

onto a single segment in $\Sigma$ which contains $b_{j}$. Continuing these two antipodal segments one direction in $\left(\Pi_{Q} \circ \Phi\right)^{-1}\{P\}$ gives antipodal paths whose final endpoints are $\left(b_{j^{\prime}}, e_{j^{\prime}}\right),\left(b_{j^{\prime}},-e_{j^{\prime}}\right)$ for some $j^{\prime}$ distinct from $j$. Here

$$
e_{j^{\prime}} \wedge e_{j^{\prime} \Sigma}=\Phi\left(b_{j^{\prime}}, \pm e_{j^{\prime}}\right)=-\Phi\left(b_{j}, \pm e_{j}\right)=-e_{j} \wedge e_{j \Sigma}
$$

Composing either antipodal path with the projection $p_{\Sigma}$ gives the same path connecting $b_{j}$ and $b_{j^{\prime}}$. Similarlly, by continuing in the other direction and projecting gives Thus the whole set

$$
B=p_{\Sigma}\left[\left(\Pi_{Q} \circ \Phi\right)^{-1}\{P\}\right]
$$

provides the desired connection in $\Sigma$.

Also note that these two paths upstairs have similar orientations induced as fibers of the map $\Pi_{Q} \circ \Phi$. That is, in the notation of slicing currents [Fe], $\S 4.3$,

$$
p_{\Sigma \#}\left\langle\llbracket \Sigma \times \mathbb{S}^{4} \rrbracket, \Pi_{Q} \circ \Phi, Q\right\rangle=2\left(\mathcal{H}^{2}\llcorner B) \wedge \vec{B}\right.
$$

where $\vec{B}$ is a unit tangent vectorfield along $B$ (in the direction running from $b_{j}$ to $b_{j^{\prime}}$ ).

\section{IV.9 Estimating the Length of the Connecting Set $B$}

The definition of $B$ depends on the choices of:

(1) the point $p \in \mathbb{S}^{3}$ near $\{0\} \times \mathbb{R}^{4}$ which gives the surface $\Sigma=u^{-1}\{p\}$ and the map

$$
\Phi:\left(\Sigma \times \mathbb{S}^{4}\right) \backslash \mathcal{N}_{\Sigma} \rightarrow \tilde{G}_{2}\left(\mathbb{R}^{5}\right), \Phi(x, e)=e \wedge e_{\Sigma}(x)
$$

(2) the 2 plane $Q \in \tilde{G}_{2}\left(\mathbb{R}^{5}\right)$ near $H$ which determines the retraction $\Pi_{\mathbb{Q}}$ of $\tilde{G}_{2}\left(\mathbb{R}^{5}\right) \backslash\{Q,-Q\}$ onto the 5 dimensional Schubert cycle $\mathcal{S}_{Q}$, and

(3) the 2 plane $P \in \mathcal{S}_{Q} \backslash \Pi_{Q}(V)$ which gives $B=p_{\Sigma}\left[\left(\Pi_{Q} \circ \Phi\right)^{-1}\{P\}\right]$. Having chosen $p \in \mathbb{S}^{3}$ as before to obtain estimate (IV.29), we need to chose $Q$ and $P$ to get the desired length estimate for $B$. 
Concerning $Q$, we first readily verify that the retraction $\Pi_{Q}$ is locally Lipschitz in $\tilde{G}_{2}\left(\mathbb{R}^{5}\right) \backslash\{Q,-Q\}$ and deduce the estimate

$$
\left|\nabla \Pi_{Q}(S)\right| \leq \frac{c}{|S-Q||S+Q|} \text { for } S \in \tilde{G}_{2}\left(\mathbb{R}^{5}\right) \backslash\{Q,-Q\} .
$$

Using (IV.43) and $\mathrm{Fe}$, 4.3.1, we may integrate the slices to find that

$$
\begin{aligned}
\int_{\mathcal{S}_{Q} \backslash \Pi_{Q}(V)} p_{\Sigma \#}\left\langle\llbracket \Sigma \times \mathbb{S}^{4} \rrbracket, \Pi_{Q} \circ \Phi, P\right\rangle d \mathcal{H}^{5} P & \leq p_{\Sigma \#} \int_{\mathcal{S}_{Q}}\left\langle\llbracket \Sigma \times \mathbb{S}^{4} \rrbracket, \Pi_{Q} \circ \Phi, P\right\rangle d \mathcal{H}^{5} P \\
& =p_{\Sigma \#}\left(\llbracket \Sigma \times \mathbb{S}^{4} \rrbracket\left\llcorner\left(\Pi_{Q} \circ \Phi\right)^{\#} \omega_{\mathcal{S}_{Q}}\right),\right.
\end{aligned}
$$

where $\omega_{\mathcal{S}_{Q}}$ is the volume element of $\mathcal{S}_{Q}$. By (IV.43) and Fatou's Lemma,

$$
\begin{aligned}
\int_{\mathcal{S}_{Q}} 2 \mathcal{H}^{1}\left(p_{\Sigma}\left[\left(\Pi_{Q} \circ \Phi\right)^{-1}\{P\}\right]\right) d \mathcal{H}^{5} P & \left.\left.=\int_{\mathcal{S}_{Q}} \mathbb{M}\left[p_{\Sigma \#}\left\langle\llbracket \Sigma \times \mathbb{S}^{4}\right]\right], \Pi_{Q} \circ \Phi, P\right\rangle\right] d \mathcal{H}^{5} P \\
& \leq \mathbb{M}\left[p _ { \Sigma \# } \left(\left[\left[\Sigma \times \mathbb{S}^{4} \rrbracket\left\llcorner\left(\Pi_{Q} \circ \Phi\right)^{\#} \omega_{\mathcal{S}_{Q}}\right)\right]\right.\right.\right. \\
& =\sup _{\alpha \in \mathcal{D}^{1}(\Sigma),|\alpha| \leq 1} \int_{\Sigma} \int_{\mathbb{S}^{4}}\left(\Pi_{Q} \circ \Phi\right)^{\#} \omega_{\mathcal{S}_{Q}} \wedge p_{\Sigma}^{\#} \alpha .
\end{aligned}
$$

To estimate this last double integral, we recall from $₫ \mathbb{I V . 3}$ that, for each fixed $x \in \Sigma \backslash\left\{a_{1}, \ldots, a_{m}\right\}$,

$$
\Phi(x, \cdot): \mathbb{S}^{4} \backslash \operatorname{Nor}(\Sigma, x) \rightarrow \mathcal{Q}_{x} \equiv \mathcal{Q}_{\operatorname{Tan}(\Sigma, x)} \backslash Y_{x}
$$

is a the smooth, orientation-preserving, 2-sheeted cover map. Each map $\Phi(x, \cdot)$ depends only on Tan $(\Sigma, x)$, and any two such maps are orthogonally conjugate. We will derive the formula

$$
\left[\left(\Pi_{Q} \circ \Phi\right)^{\#} \omega_{\mathcal{S}_{Q}} \wedge p_{\Sigma}^{\#} \alpha\right](x, \cdot)=\beta(x, \cdot) p_{\Sigma}^{\#} \omega_{\Sigma}(x) \wedge \Phi(x, \cdot)^{\#} \omega_{\mathcal{Q}_{x}}
$$

where $\omega_{\Sigma}$ and $\omega_{\mathcal{Q}_{x}}$ denote the volume elements of $\Sigma$ and $\mathcal{Q}_{x}$ and $\beta(x, \cdot)$ is a smooth function on $\mathbb{S}^{4} \backslash$ $\operatorname{Nor}(\Sigma, x)$ satisfying

$$
|\beta(x, e)| \leq \frac{c}{|\Phi(x, e)-Q|^{5}|\Phi(x, e)+Q|^{5}} \sum_{j=1}^{3}\left|\nabla \tilde{\tau}_{j}(x)\right| \text { for } e \in \mathbb{S}^{4} .
$$

Before proving (IV.46), note that the decomposition on the righthand side is not necessarily smooth in $x$ since the different $\mathcal{Q}_{x}$ may overlap for $x$ near a critical point of $\Phi(\cdot, e)$ for some $e \in \mathbb{S}^{4}$. Nevertheless, the formula does imply the measurability of $\beta(x, e)$ in $x$, and so may be integrated over $\Sigma$.

To derive (IV.46), we first note that, with the factorization $\Sigma \times \mathbb{S}^{4}$, there are only two terms in the $(p, q)$ decomposition of the 5 form,

$$
\left(\Pi_{Q} \circ \Phi\right)^{\#} \omega_{\mathcal{S}_{Q}}=\Omega_{2,3}+\Omega_{1,4} .
$$

Thus,

$$
\left(\Pi_{Q} \circ \Phi\right)^{\#} \omega_{\mathcal{S}_{Q}} \wedge p_{\Sigma}^{\#} \alpha=0+\Omega_{1,4} \wedge p_{\Sigma}^{\#} \alpha
$$

because the term $\Omega_{2,3} \wedge p_{\Sigma}^{\#} \alpha$, being of type $(2+1,3)$, must vanish.

For each $S=\Phi(x, \pm e) \in \mathcal{Q}_{x} \backslash Y_{x}$, we also have the factorization

$$
\operatorname{Tan}\left(\tilde{G}_{2}\left(\mathbb{R}^{5}\right), S\right)=\operatorname{Nor}\left(\mathcal{Q}_{x}, S\right) \times \operatorname{Tan}\left(\mathcal{Q}_{x}, S\right) .
$$


Let $\mu_{1}, \mu_{2}, \mu_{3}, \mu_{4}, \nu_{1}, \nu_{2}$ be an orthonormal basis of $\wedge^{1} \operatorname{Tan}\left(\tilde{G}_{2}\left(\mathbb{R}^{5}\right), S\right)$ so that

$$
\mu_{1}, \mu_{2}, \mu_{3}, \mu_{4} \in \wedge^{1} \operatorname{Tan}\left(\mathcal{Q}_{x}, S\right), \nu_{1}, \nu_{2} \in \wedge^{1} \operatorname{Nor}\left(\mathcal{Q}_{x}, S\right), \mu_{1} \wedge \mu_{2} \wedge \mu_{3} \wedge \mu_{4}=\omega_{\mathcal{Q}_{x}}(S) ;
$$

thus, $0=\nu_{1}(v)=\nu_{2}(v)=\mu_{1}(w)=\mu_{2}(w)=\mu_{3}(w)=\mu_{4}(w)$ whenever $v \in \operatorname{Tan}\left(\mathcal{Q}_{x}, S\right)$ and $w \in$ $\operatorname{Nor}\left(\mathcal{Q}_{x}, S\right)$. We may expand the 5 covector

$$
\begin{aligned}
\Pi_{Q}^{\#}\left(\omega_{\mathcal{S}_{Q}}\right)(S)= & \lambda_{1} \nu_{2} \wedge \mu_{1} \wedge \mu_{2} \wedge \mu_{3} \wedge \mu_{4}+\lambda_{2} \nu_{1} \wedge \mu_{1} \wedge \mu_{2} \wedge \mu_{3} \wedge \mu_{4}+\lambda_{3} \nu_{1} \wedge \nu_{2} \wedge \mu_{2} \wedge \mu_{3} \wedge \mu_{4} \\
& +\lambda_{4} \nu_{1} \wedge \nu_{2} \wedge \mu_{1} \wedge \mu_{3} \wedge \mu_{4}+\lambda_{5} \nu_{1} \wedge \nu_{2} \wedge \mu_{1} \wedge \mu_{2} \wedge \mu_{4}+\lambda_{6} \nu_{1} \wedge \nu_{2} \wedge \mu_{1} \wedge \mu_{2} \wedge \mu_{3}
\end{aligned}
$$

where

$$
\left|\lambda_{i}\right| \leq \frac{c}{|S-Q|^{5}|S+Q|^{5}}
$$

by (IV.44). Applying $\Phi^{\#}$ (that is, $\left.\wedge^{1} D \Phi(x, e)\right)$ to all covectors and taking the $(1,4)$ component, we find that only the first two terms survive so that

$$
\begin{aligned}
\Omega_{1,4}(x, e) & =\left[\lambda_{1} \Phi^{\#} \nu_{2}+\lambda_{2} \Phi^{\#} \nu_{1}\right]_{(1,0)} \wedge \Phi^{\#} \mu_{1} \wedge \Phi^{\#} \mu_{2} \wedge \Phi^{\#} \mu_{3} \wedge \Phi^{\#} \mu_{4} \\
& =\left[\lambda_{1} \Phi^{\#} \nu_{2}+\lambda_{2} \Phi^{\#} \nu_{1}\right]_{(1,0)} \wedge \Phi(x, \cdot)^{\#} \omega_{\mathcal{Q}_{x}}(S) .
\end{aligned}
$$

Being of type $(2,0)$, the 2 covector

$$
\left(\left[\lambda_{1} \Phi^{\#} \nu_{2}+\lambda_{2} \Phi^{\#} \nu_{1}\right]_{1,0} \wedge p_{\Sigma}^{\#} \alpha\right)(x, e)=\beta(x, e) p_{\Sigma}^{\#} \omega_{\Sigma}(x)
$$

for some scalar $\beta(x, e)$, and (IV.48), (IV.50), and (IV.51) now give the desired formula (IV.46). This formula readily implies the smoothness of $\beta(x, \cdot)$ on $\mathbb{S}^{4} \backslash \operatorname{Nor}(\Sigma, x)$.

To verify the bound (IV.47), observe that

$$
\left|\left[\Phi^{\#} \nu_{i}\right]_{1,0}\right|=\sup _{v \in \mathbb{S}^{4} \cap \operatorname{Tan}(\Sigma, x)} \nu_{i}\left[\nabla_{v} \Phi(x, e)\right]
$$

where $\nabla_{v} \Phi(x, e)=D \Phi_{(x, e)}(v, 0) \in \operatorname{Tan}\left(\tilde{G}_{2}\left(\mathbb{R}^{5}\right), S\right)$. For any unit vector $v \in \operatorname{Tan}(\Sigma, x)$ and any $w \in \mathbb{R}^{5}$,

$$
v \wedge w \in \operatorname{Tan}\left(\mathcal{Q}_{x}, S\right)
$$

because we may assume $w \notin \operatorname{Tan}(\Sigma, x)$ and then choose a curve $y(t)$ in $\mathbb{S}^{4} \cap v^{\perp} \backslash \operatorname{Nor}(\Sigma, x)$ with $y^{\prime}(0)=$ $w-(w \cdot v) v$, hence,

$$
v \wedge w=v \wedge y^{\prime}(0)=\frac{d}{d t}_{t=0}(v \wedge y(t))=-\frac{d}{d t}_{t=0} \Phi(x, y(t)) .
$$

Thus, for any 2 vector $\xi \in \operatorname{Nor}\left(\mathcal{Q}_{x}, S\right),|\xi|=|\xi \wedge v| ;$ in particular, $|\xi|=\left|\xi \wedge \tilde{e}_{T}(x)\right|,|\xi|=\left|\xi \wedge e_{\Sigma}(x)\right|$, and hence,

$$
|\xi|=\left|\xi \wedge\left(\tilde{e}_{T}(x) \wedge e_{\Sigma}(x)\right)\right| .
$$

Since $\nu_{i} \in \wedge^{1} \operatorname{Nor}\left(\mathcal{Q}_{x}, S\right)$ and $\left|\nu_{i}\right|=1$, we now find that

$$
\nu_{i}\left[\nabla_{v} \Phi(x, e)\right]=\nu_{i}\left[\left(\nabla_{v} \Phi(x, e)\right)_{\operatorname{Nor}(\Sigma, x)}\right] \leq\left|\nabla_{v} \Phi(x, e) \wedge\left(\tilde{e}_{T}(x) \wedge e_{\Sigma}(x)\right)\right| .
$$

Moreover,

$$
\begin{aligned}
\left|\nabla_{v} \Phi \wedge\left(\tilde{e}_{T} \wedge e_{\Sigma}\right)\right| & \leq\left|\left(\nabla_{v}\left(e \wedge e_{\Sigma}\right)\right) \wedge\left(\tilde{e}_{T} \wedge e_{\Sigma}\right)\right| \leq\left|\left(\nabla_{v} e_{\Sigma}\right) \wedge\left(\tilde{e}_{T} \wedge e_{\Sigma}\right)\right| \\
& =\left|e_{\Sigma} \wedge \nabla_{v}\left(\tilde{e}_{T} \wedge e_{\Sigma}\right)\right| \leq\left|\nabla_{v}\left(\tilde{e}_{T} \wedge e_{\Sigma}\right)\right| \\
& =\left|\nabla_{v}\left(*\left(\tilde{\tau}_{1} \wedge \tilde{\tau}_{2} \wedge \tilde{\tau}_{3}\right)\right)\right| \leq c \sum_{j=1}^{3}\left|\nabla \tilde{\tau}_{j}\right|
\end{aligned}
$$


where $*$ is the Hodge $*: \wedge_{3} \mathbb{R}^{5} \rightarrow \wedge_{2} \mathbb{R}^{5} \approx \mathbb{R}^{5}$ [F,1.7.8]) The desired pointwise bound (IV.47) now follows by combining (IV.49), (IV.51), (IV.52), (IV.53) and (IV.54).

For each $x \in \Sigma$, the pull-back $\Phi(x, \cdot)^{\#} \omega_{\mathcal{Q}_{x}}$ is point-wise a positive multiple of the volume form of $\mathbb{S}^{4}$. So we may first integrate over $\mathbb{S}^{4}$ and use (IV.47) to see that

$$
\begin{aligned}
\int_{\mathbb{S}^{4}} \beta(x, \cdot) \Phi(x, \cdot)^{\#} \omega_{\mathcal{Q}_{x}} & \leq \int_{\mathbb{S}^{4}}|\beta(x, \cdot)| \Phi(x, \cdot)^{\#} \omega_{\mathcal{Q}_{x}} \\
& \leq c\left(\sum_{j=1}^{3}\left|\nabla \tilde{\tau}_{j}(x)\right|\right) \int_{\mathbb{S}^{4}} \frac{\Phi(x, \cdot)^{\#} \omega_{\mathcal{Q}_{x}}}{|\Phi(x, \cdot)-Q|^{5}|\Phi(x, \cdot)+Q|^{5}} \\
& =c\left(\sum_{j=1}^{3}\left|\nabla \tilde{\tau}_{j}(x)\right|\right) \int_{\mathcal{Q}_{x}} \frac{\omega_{\mathcal{Q}_{x}}(S)}{|S-Q|^{5}|S+Q|^{5}} \\
& \leq c\left(\sum_{j=1}^{3}\left|\nabla \tilde{\tau}_{j}(x)\right|\right) \int_{\mathcal{Q}_{x}} \frac{d \mathcal{H}^{4} S}{|S-Q|^{5}|S+Q|^{5}} .
\end{aligned}
$$

To handle the denominator, we note that the Grassmannian $\tilde{G}_{2}\left(\mathbb{R}^{5}\right)$ is a 6 dimensional homogeneous space, and we readily use local coordinates to verify that

$$
C_{3}=\int_{\tilde{G}_{2}\left(\mathbb{R}^{5}\right)} \frac{1}{|S-Q|^{5}|S+Q|^{5}} d \mathcal{H}^{6} Q<\infty
$$

independent of $S$.

Now we recall (IV.45) and fix a sequence of 1 forms $\alpha_{i} \in \mathcal{D}^{1}(\Sigma)$ with $\left|\alpha_{i}\right| \leq 1$ so that

$$
\mathbb{M}\left[p_{\Sigma \#}\left(\llbracket \Sigma \times \mathbb{S}^{4} \rrbracket \mathbf{L}\left(\Pi_{Q} \circ \Phi\right)^{\#} \omega_{\mathcal{S}_{Q}}\right)\right]=\lim _{i \rightarrow \infty} \int_{\Sigma} \int_{\mathbb{S}^{4}}\left(\Pi_{Q} \circ \Phi\right)^{\#} \omega_{\mathcal{S}_{Q}} \wedge p_{\Sigma}^{\#} \alpha_{i},
$$

let $\beta_{i}$ be the corresponding function from the formula (IV.46), and use (IV.45), Fatou's Lemma, (IV.46), (IV.55), Fubini's Theorem, (IV.56), and (IV.29) to obtain our final integral estimate

$$
\begin{aligned}
& \int_{\tilde{G}_{2}\left(\mathbb{R}^{5}\right)} \int_{\mathcal{S}_{Q}} 2 \mathcal{H}^{1}\left(p_{\Sigma}\left[\left(\Pi_{Q} \circ \Phi\right)^{-1}\{P\}\right]\right) d \mathcal{H}^{5} P d \mathcal{H}^{6} Q \\
& \leq \int_{\tilde{G}_{2}\left(\mathbb{R}^{5}\right)} \lim _{i \rightarrow \infty} \int_{\Sigma} \int_{\mathbb{S}^{4}}\left(\Pi_{Q} \circ \Phi\right)^{\#} \omega_{\mathcal{S}_{Q} \backslash \Pi_{Q}(V)} \wedge p_{\Sigma}^{\#} \alpha_{i} \\
& \leq \liminf _{i \rightarrow \infty} \int_{\tilde{G}_{2}\left(\mathbb{R}^{5}\right)} \int_{\Sigma} \int_{\mathbb{S}^{4}} \beta_{i}(x, \cdot) \omega_{\Sigma}(x) \wedge \Phi(x, \cdot)^{\#} \omega_{\mathcal{Q}_{x}} \\
& \leq c \int_{\Sigma}\left(\sum_{j=1}^{3}\left|\nabla \tilde{\tau}_{j}(x)\right|\right) \int_{\mathcal{Q}_{x}} \int_{\tilde{G}_{2}\left(\mathbb{R}^{5}\right)} \frac{1}{|S-Q|^{5}|S+Q|^{5}} d \mathcal{H}^{6} Q d \mathcal{H}^{4} S d \mathcal{H}^{2} x \\
& \leq c C_{3} \sum_{j=1}^{3} \int_{\Sigma}\left|\nabla \tilde{\tau}_{j}(x)\right| d \mathcal{H}^{2} x \leq c \int_{\mathbb{B}^{5}}\left|\nabla^{2} u\right|^{2} d x .
\end{aligned}
$$

The Schubert cycles $\mathcal{S}_{Q}$ are all orthogonally equivalent and have the same positive 5 dimensional Hausdorff measure. So we can use the final integral inequality to choose first a 2 plane $Q \in \tilde{G}_{2}\left(\mathbb{R}^{5}\right)$ and then a 2 plane $P \in \mathcal{S}_{Q}$ so that the corresponding connecting set

$$
B=p_{\Sigma}\left[\left(\Pi_{Q} \circ \Phi\right)^{-1}\{P\}\right]
$$


satisfies the desired length estimate

$$
\mathcal{H}^{1}(B) \leq c \int_{\mathbb{B}^{5}}\left|\nabla^{2} u\right|^{2} d x
$$

Theorem IV.2 (Length Bound) For any $u \in \mathcal{R}$, Singu has a $\mathbb{Z}_{2}$ connection $\Gamma$ satisfying

$$
\mathcal{H}^{1}(\Gamma) \leq c \int_{\mathbb{B}^{5}}\left|\nabla^{2} u\right|^{2} d x
$$

for some absolute constant $c$.

Proof. To form the connection $\Gamma$, one takes the union of the curves from $A$ and $B$. The behavior of the individual curves near the points $a_{i}$ and $b_{j}$ has been discussed in subsection IV.5. The set $A \cup B$ will pass through each point $b_{j}$, likely having a corner at $b_{j}$. One easily replaces the corner with an embedded smooth curve near $b_{j}$. Also the curves contributing to $A$ and $B$ may cross. In $\mathbb{B}^{5}$, it is easy to perturb the curves to eliminate such crossings. The result is the desired $\mathbb{Z}_{2}$ connection $\Gamma$. (Alternately one can observe that $A \cup B$ already defines a one dimensional integer multiplicity chain modulo 2 (see [Fe] [4.2.26] which has boundary $\sum_{i=1}^{m} \llbracket a_{i} \rrbracket$ relative to $\partial \mathbb{B}^{5}$. As mentioned before, the minimal (mass-minimizing) connection will automatcally consist of non-overlapping intervals. Those that reach $\partial \mathbb{B}^{5}$ meet it orthogonally.

\section{Sequential Weak Density of $W^{2,2}\left(\mathbb{B}^{5}, \mathbb{S}^{3}\right)$}

We are now ready to prove:

Theorem V.3 Any map $v$ in $W^{2,2}\left(\mathbb{B}^{5}, \mathbb{S}^{3}\right)$ may be approximated in the $W^{2,2}$ weak topology by a sequence of smooth maps.

Proof. First we may, by Lemma III.2, chose, for each positive integer $i$, a map $u_{i} \in \mathcal{R}$ so that $\| u_{i}-$ $v \|_{W^{2,2}}<\frac{1}{i} ;$ in particular,

$$
\left\|u_{i}-v\right\|_{L^{2}}<\frac{1}{i} \text { and } I=\sup _{i} \int_{\mathbb{B}^{5}}\left|\nabla^{2} u_{i}\right|^{2} d x<\infty
$$

Applying Lemma III.1 to each $u_{i}$, we note that, as $\varepsilon \rightarrow 0$, the smooth approximates $u_{i, \varepsilon}$ approach $u_{i}$ pointwise on $\mathbb{B}^{5} \backslash \operatorname{Sing} u_{i}$. Inasmuch as the $u_{i, \varepsilon}$ are pointwise bounded (by 1), Lebesgue's theorem implies

$$
\left\|u_{i, \varepsilon}-u_{i}\right\|_{L^{2}} \rightarrow 0 \quad \text { as } \quad \varepsilon \rightarrow 0
$$

Thus we can choose a positive $\varepsilon_{i}$, so that the smooth map $w_{i}=u_{i, \varepsilon_{i}}$ has $\left\|w_{i}-u_{i}\right\|_{L^{2}}<\frac{1}{i}$; in particular, $\left\|w_{i}-v\right\|_{L^{2}}<\frac{2}{i}$, and the smooth maps $w_{i}$ converge to $v$ strongly in $L^{2}$.

On the other hand, by Lemma II.1, Lemma II.1, Theorem IV.2, and (V),

$$
\sup _{i}\left\|w_{i}\right\|_{W^{2,2}}^{2}<2 c_{m}(1+I)<\infty
$$

By the weak* (=weak) compactness of the closed ball in $W^{2,2}\left(\mathbb{B}^{5}, \mathbb{R}^{\ell}\right)$, the sequence $w_{i}$ contains a subequence $w_{i^{\prime}}$ that is $W^{2,2}$ weakly convergent to some $w \in W^{2,2}\left(\mathbb{B}^{5}, \mathbb{R}^{\ell}\right)$. But, $w$, being by Rellich's theorem, the strong $L^{2}$ limit of the $w_{i^{\prime}}$, must necessarily be the original map $v$. 


\section{V.1 Least Connection Length $L(v)$}

By Lemma III.2 one may now define, for any Sobolev map $v \in W^{2,2}\left(\mathbb{B}^{5}, \mathbb{S}^{3}\right)$, the nonnegative number $L(v)=\lim _{\varepsilon \rightarrow 0} \inf \left\{\mathcal{H}^{1}(\Gamma): \Gamma\right.$ is a $\mathbb{Z}_{2}$ connection for Sing $u$ for some $u \in \mathcal{R}$ with $\left.\|u-v\|_{W^{2,2}}<\varepsilon\right\}$.

Any $u \in \mathcal{R}$ has a minimal $\mathbb{Z}_{2}$ connection, and $L(u)$ is its length. In general:

Theorem V.4 For any $v \in W^{2,2}\left(\mathbb{B}^{5}, \mathbb{S}^{3}\right), L(v)=0 \Longleftrightarrow v$ is the $W^{2,2}$ strong limit of smooth maps.

Proof. The sufficiency is immediate from the definition of $L(v)$. To prove the necessity, we assume $L(v)=0$. Then we may choose, for each $i$, a map $u_{i} \in \mathcal{R}$ along with a $\mathbb{Z}_{2}$ connection $\Gamma_{i}$ of $\operatorname{Sing} u_{i}$ so that $\left\|u_{i}-v\right\|_{W^{2,2}}<1 / i$ and $\mathcal{H}^{1}\left(\Gamma_{i}\right)<1 / i$. As in the previous proof, there is an $\varepsilon_{i}<1 / i$ so that the smooth maps $w_{i}=u_{i, \varepsilon_{i}}$ converge strongly in $L^{2}$ and weakly in $W^{2,2}$ to $v$. The lower-semicontinuity

$$
\int_{\mathbb{B}^{5}}\left|\nabla^{2} v\right|^{2} d x \leq \liminf _{i \rightarrow \infty} \int_{\mathbb{B}^{5}}\left|\nabla^{2} w_{i}\right|^{2} d x
$$

follows. On the other hand, we have from Theorem $\amalg I .1$ the inequality

$$
\int_{\mathbb{B}^{5}}\left|\nabla^{2} w_{i}\right|^{2} d x-\int_{\mathbb{B}^{5}}\left|\nabla^{2} u_{i}\right|^{2} d x \leq \frac{1}{i}+\frac{c_{\mathbb{S H}}}{i} .
$$

as well as, from Lemma 11.2 the $W^{2,2}$ strong convergence

$$
\lim _{i \rightarrow \infty} \int_{\mathbb{B}^{5}}\left|\nabla^{2} u_{i}\right|^{2} d x=\int_{\mathbb{B}^{5}}\left|\nabla^{2} v\right|^{2} d x
$$

which together imply the upper semi-continuity

$$
\int_{\mathbb{B}^{5}}\left|\nabla^{2} v\right|^{2} d x \geq \limsup _{i \rightarrow \infty} \int_{\mathbb{B}^{5}}\left|\nabla^{2} w_{i}\right|^{2} d x .
$$

The convergence of the total Hessian energies of the $w_{i}$ to that of $v$, along with the $W^{2,2}$ weak convergence, now implies the $W^{2,2}$ strong convergence of the smooth maps $w_{i}$ to $v$.

\section{References}

[A] Adams. "Sobolev Functions". Academic Press, New York, 1974.

[ABL] F. Almgren, W. Browder, E.H. Lieb. "Co-area, liquid crystals, and minimal surfaces". Partial Differential Equations (Tianjin 1986), Lecture Notes in Mathematics, 1306, Springer, Berlin, 1988.

[ABO] G. Alberti, S. Baldo, and G. Orlandi. "Functions with prescribed singularities". J. Eur. Math. Soc. (JEMS), 5 (2003), 275-311.

[Be1] F. Bethuel. "A characterization of maps in H1(B3,S2) which can be approximated by smooth maps". Ann. Inst. H. Poincar Anal. Non Linéaire 7 (1990), no. 4, 269-286.

[Be2] F. Bethuel. "The approximation problem for Sobolev maps between two manifolds". Acta Math. 167(1991), no.3-4, 153-206.

[BBC] F. Bethuel, H. Brezis, and J.-M. Coron, Relaxed energies for harmonic maps. Variational methods, 37-52, Progr. Nonlin. Diff. Eqns. Appl.,4, Birkhuser Boston 1990. 
[BCDH] F. Bethuel, J.-M. Coron, F. Demengel, F. Hlein A cohomological criterion for density of smooth maps in Sobolev spaces between two manifolds. Nematics (Orsay, 1990), 15-23, NATO Adv. Sci. Inst. Ser. C Math. Phys. Sci., 332, Kluwer Acad. Publ., Dordrecht, 1991.

[BCL] H. Brezis, J.-M.Coron, and E. Lieb, "Harmonic maps with defects". Comm. Math. Phys., 107 (1986), 649-705.

[BZ] F. Bethuel and X.M. Zheng. Density of smooth functions between two manifolds in Sobolev spaces. J. Funct. Anal. 80 (1988), no. 1, 60-75.

[BPV] P. Bousquet, A. Ponce, and J. Van Schaftigen. " Strong Density for Higher Order Sobolev Spaces into Compact Manifolds", arXiv:1203.3721.

[Br] G. Bredon. "Topology and Geometry", Graduate Texts in Mathematics, Springer 1993.

[Fe] H. Federer. "Geometric Measure Theory", Springer 1969.

[GMS1] M. Giaquinta, G. Modica, and J. Souček. "The Dirichlet energy of mappings with values into the sphere". Manuscripta Math., 65 (1989), 489-507.

[GMS2] M. Giaquinta, G. Modica, and J. Souček. "Cartesian Currents in the Calculus of Variations I, II". Ergebnisse der Mathematik und ihrer Grenzgebiete. A Series of Modern Surveys in Mathematics, 37, 38. Springer, Berlin-Heidelberg, 1998.

[Ha] F. Hang. "On the weak limits of smooth maps for the Dirichlet energy between manifolds". Comm. Anal. Geom., 13 (2005), no. 5, 929-938.

[HaL1] F. Hang and F.H. Lin. "Topology of Sobolev mappings". Math. Res. Lett. 8 (2001), no. 3, 321-330.

[HaL2] F. Hang and F.H. Lin. "Topology of Sobolev mappings". II. Acta Math. 191 (2003), no. 1, 55107.

[HL] R. Hardt and F.H. Lin. "A remark on H1 mappings". Manuscripta Math. 56 (1986), no. 1, 1-10.

[HR] R. Hardt and T. Rivière. "Connecting topological Hopf singularities", Ann. Sc. Norm. Super. Pisa Cl. Sci. (5)2 (2003), no. 2, 287-344.

[Hj] P. Hajlasz. "Approximation of Sobolev mappings". Nonlinear Anal. 22 (1994), no. 12, 1579-1591.

[MS] J. Milnor and J. Stasheff. "Characteristic Classes" Princeton University Press, 1974.

[MR] T. Rivière and Y. Meyer. "Partial Regularity for a class of stationary Yang-Mills Fields", Rev. Math. Iberoamericana, 19(2003), 195-219.

[Pa] M.R. Pakzad "Weak density of smooth maps in $W^{1,1}(M, N)$ for non-abelian $\pi_{1}(N)$ ". Ann. Global Anal. Geom. 23 (2003), no. 1, 112.

[PR] M. R. Pakzad and T. Rivière. "Weak density of smooth maps for the Dirichlet energy between manifolds". Geom. Funct. Anal. 13 (2003), no. 1, 223-257.

[Ri] T. Rivière, "Sobolev critical exponents of rational homotopy groups." Pure Appl. Math. Q. 3 (2007), no. 2, Special Issue: In honor of Leon Simon. Part 1, 615630.

[SU] R. Schoen and K. Uhlenbeck. "Approximation theorems for Sobolev mappings", Preprint (1984).

[W] B. White. "Homotopy classes in Sobolev spaces and the existence of energy minimizing maps". Acta. Math., 160 (1988), 1-17. 